\title{
Asymptotic analysis of breather modes in a two-dimensional mechanical lattice
}

\author{
Jonathan AD Wattis ${ }^{1 *}$ and Ahmed SM Alzaidi ${ }^{2,3}$ \\ ${ }^{1}$ School of Mathematical Sciences, University of Nottingham, \\ University Park, Nottingham, NG7 2RD, UK. \\ ${ }^{2}$ School of Computing and Mathematics, Keele University, \\ Staffordshire, ST5 5BG, UK. \\ ${ }^{3}$ Department of Mathematics and Statistics, Taif University, \\ P.O.Box 888, Taif, Saudi Arabia.
}

\begin{abstract}
We consider a two-dimensional square lattice in which each node is restricted to the plane of the lattice, but is permitted to move in both directions of the lattice. We assume nodes are connected to nearest neighbours along the lattice directions with nonlinear springs, and to diagonal neighbours with linear springs. We consider a generalised Klein-Gordon system, that is, where there is an onsite potential at each node in addition to the (nonlinear) nearest-neighbour interactions. We derive the equations of motion for the displacements from the Hamiltonian. We use asymptotic techniques to derive the form of small amplitude breather solutions, and find necessary conditions required for their existence. We find two types of mode, which we term 'optical' and 'acoustic', based on the analysis of other lattices which support dispersion relations with multiple branches. In addition to the usual inequality on the sign of the nonlinearity in order for the NLS to be of the focusing type, we obtain an additional ellipticity constraint, that is a restriction in the two-dimensional wavenumber space, required for the spatial differential operator to be elliptic.
\end{abstract}

\section{Highlights}

- we consider a 2D square lattice with in-plane motion of nodes

- we use a weakly nonlinear asymptotic expansion to derive envelope equation

- we find breather solutions of an associated 2D NLS

- two conditions for breathers: usual focusing, additional ellipticity constraint

Key words: breathers; nonlinear waves; discrete systems; asymptotic analysis

Email address: Jonathan. Wattis@nottingham.ac.uk a.s.m.alzaidi@keele.ac.uk (Jonathan AD Wattis ${ }^{1 *}$ and Ahmed SM Alzaidi ${ }^{2,3}$ ). 


\section{Introduction}

In this paper, we investigate breathers in two-dimensional mechanical lattices. Discrete breathers are spatially localised waves which have an internal oscillatory mode. The simplest way to visualise of these systems is a lattice of particles confined to a plane. At each node, the particle can move in the plane, that is, it has two degrees of freedom, with displacement vectors written in terms of the direction vectors of the lattice. The discreteness of the lattice causes the dispersion relation for linear modes to have a finite range, giving rise to frequencies where only nonlinear modes can exist. The form of dispersion relations in such lattices is frequently derived by those studying this type of system, and is easily obtained from a small amplitude weakly nonlinear expansion. The novelty of our approach lies in solving the asymptotic expansion at higher orders, in a system where the displacements in the two directions are coupled. We obtain the amplitude of second harmonic terms for both displacements, and an equation governing the shape of the envelope solution. We find two conditions for breather solutions to exist: first, the usual condition in order for the 'focusing' form of the NLS to be obtained; and a second 'ellipticity' constraint, which does not occur in the one-dimensional case. Here, we only consider Hamiltonian systems, that is, conservative systems where there are no losses due to friction or damping, etc.

The motivation to study such systems comes from understanding how energy is transported through crystal structures, one example of which is the use of metal barriers to shield radiation. We are interested in whether such crystal lattice structures could transport localised pulses of energy without dispersion or dissipation. If radiation could initiate dislocations and cause them to accumulate, then the physical properties of the shield could be affected, potentially resulting in undesirable deleterious effects on the integrity of the shield. Discrete breathers have also been postulated as a mechanism for high Temperature superconductivity [23], and are of interest in the study of granular crystals [21]. The behaviour of breather modes in discrete nonlinear systems has been widely studied, under the terms 'nonlinear localised excitations', 'intrinsic localised modes', or more commonly in recent years, 'discrete breathers'.

Over the last few decades, many studies of discrete breathers have been published. From the start, many general results were found to be valid for high dimensional lattices as well as one-dimensional (1D) chains. For example, Takeno [29] applied Green's function techniques to find analytical approximations of breather solutions in one-, two- and three-dimensional (3D) lattices. The initial proofs of existence of breathers by MacKay and Aubry [22] relied on the anticontinuum limit, in which the coupling between neighbouring nodes is vanishingly small. Such techniques are as applicable in two and three dimen- 
sions as in one dimension. However, the energy-amplitude relationship differs dramatically with dimension: in 1D, the energy of breather decreases to zero with the amplitude, whereas in 3D, as the amplitude decreases to zero, the energy increases without bound, as shown by Flach [17].

The explicit calculation of approximate breather modes was initially attempted in one-dimensional systems, where the asymptotic reduction to NLS was found, see, for example, Remoissenet [27]. Numerical approximations for two-dimensional (2D) systems were obtained by Marin et al. [23], who initially considered the case of the triangular lattice with both nonlinear nearest-neighbour interactions alongside a nonlinear onsite potential. The same authors later considered diatomic square lattice [24], once again finding moving breathers.

Until recently, the theoretical analysis of two-dimensional lattices has lagged behind numerical works. A good recent review of the theory of two-dimensional lattices can be obtained from the book of Archilla et al. [1]. The second chapter, by Bajars et al. [2] describes various phenomena observed in simulations of two-dimensional lattices with displacements in both in-plane directions. Whilst this work covers plane-wave kink-solutions, the main focus is on moving breathers which are localised in both spatial dimensions. Whilst such modes do not propagate in a dispersionless manner, they are extremely long-lived, and in many simulations, show remarkable robustness when colliding with each other. Simulations of kinks - a dislocation due to a vacancy - also move through the lattice with very little loss in energy; however, when they meet another dislocation, they produce stationary and moving breathers. Details of the loss in energy and reduction in breather speed is given in the paper of Bajars et al. [3].

Related work on two-dimensional systems where solitary waves are relevant includes Besse et al. [5, 6], who summarise the behaviour of dipoles and vortices in the two-dimensional Ginzburg-Landau equation with cubic-quintic nonlinearities. In [12], Cisneros-Ake and Minzoni analyse lateral dispersion in discrete and continuous two-dimensional models using the KadomtsevPetviashvili equation. They deduce connections between impurities and imposed stress produce solutions analogous to soliton modes, and draw analogies with the work of Smyth [28] on resonant flow over prescribed topography, and Chetverikov et al. [10] who analyse a 2D system modelling electron motion in a crystal lattice. The Peierls-Nabarro (PN) potential has been used extensively in analysing the mobility of travelling waves in discrete one-dimensional systems. This concept has been generalised by Vicencio and Johannson [30] to two-dimensional lattices. In [19], Johannson and Jason extend these ideas to breathers in discrete 2D NLS, finding a PN potential which depends on the direction of motion through the lattice. The idea of a PN potential has also been generalised to cover breathers in one-dimensional systems [32], where it is found to depend on the internal phase of the breather. Dmitriev et al. [14] 
simulate breathers in 2D and 3D systems with FCC, BCC, and HCP geometry, generally finding that breathers are robust.

We have previously considered two-dimensional electrical transmission lattices composed of inductors and nonlinear capacitors, in which there is only a scalar unknown, the charge $Q_{m, n}(t)$, at each lattice site $(m, n)$ [31], covering the square [8], triangular [9] and honeycomb arrangements [33]. These cases are considerably simpler than the mechanical system considered by Marin et al. $[23,24]$, in which a two-component vector has to be found at each node, and in such systems, the unknown horizontal and vertical displacements are intrinsically coupled together. From numerical simulations of Marin et al. [23, 24], Yi et al. [34] noted that moving breathers are narrow in the direction perpendicular to their motion, and so simplified the governing equations to a 'triple ladder' model, finding a system of coupled NLS equations in $1+1$ dimensions, which supported both stationary and moving breathers.

In this paper, we follow Marin et al. [23, 24], and include both nonlinear onsite potential and nonlinear nearest-neighbour interactions. In one-dimension, the inclusion of nonlinear rather than linear nearest-neighbour interactions is a significant complication; however, in two-dimensional systems, even system with linear interactions generate nonlinear equations of motion due to the geometry of the lattice. In the remainder of this section, we summarise the formulation of Klein-Gordon and Fermi-Pasta-Ulam systems in one-dimension, before addressing two-dimensional Klein-Gordon problems in the remainder of the paper. The two-dimensional FPU system will be the topic of a subsequent paper. In Section 2 we formulate the KG problem using Hamiltonian mechanics, and in Section 3 we detail the asymptotic reduction which leads to a nonlinear Schrödinger system. The work is summarised and conclusions drawn in Section 4. The paper concludes with an appendix where details of the lengthier expressions used in Sections 2 and 3 are quoted, and a couple of special cases are illustrated.

\subsection{One-dimensional systems}

In one-dimension, the governing equation for a general lattice with nearestneighbour interactions is

$$
\frac{\mathrm{d}^{2} u_{n}}{\mathrm{~d} t^{2}}=V^{\prime}\left(u_{n+1}-u_{n}\right)-V^{\prime}\left(u_{n}-u_{n-1}\right)-V_{0}^{\prime}\left(u_{n}\right),
$$

which arises from the Hamiltonian

$$
H=\sum_{n} \frac{1}{2}\left(\frac{\mathrm{d} u_{n}}{\mathrm{~d} t}\right)^{2}+V_{0}\left(u_{n}\right)+V\left(u_{n+1}-u_{n}\right) .
$$


Most analysis of such lattice equations usually focuses on two distinct simplifications: namely

- Klein-Gordon (KG) lattices, in which $V^{\prime}(\phi)=k \phi$, that is, nearest-neighbour interactions are linear, and there is a nonlinear onsite potential, $V_{0}$, leading to the governing equation

$$
\frac{\mathrm{d}^{2} u_{n}}{\mathrm{~d} t^{2}}=k\left(u_{n+1}-2 u_{n}+u_{n-1}\right)-V_{0}^{\prime}\left(u_{n}\right) .
$$

- Fermi-Pasta-Ulam (FPU) lattices, in which there is no onsite potential ( $V_{0}=$ $0)$ and the nearest-neighbour interactions $\left(V^{\prime}(\phi)\right)$ are nonlinear, hence

$$
\frac{\mathrm{d}^{2} u_{n}}{\mathrm{~d} t^{2}}=V^{\prime}\left(u_{n+1}-u_{n}\right)-V^{\prime}\left(u_{n}-u_{n-1}\right) .
$$

Using $\phi_{n}=u_{n+1}-u_{n}$, this equation can be rewritten as

$$
\frac{\mathrm{d}^{2} \phi_{n}}{\mathrm{~d} t^{2}}=V^{\prime}\left(\phi_{n+1}\right)-2 V^{\prime}\left(\phi_{n}\right)+V^{\prime}\left(\phi_{n-1}\right)
$$

which simplifies the analysis and asymptotic derivation of solutions of the FPU lattice [7, 18].

However, in two-dimensional lattices, even harmonic nearest neighbour interactions (i.e. quadratic) potentials generate forces which are nonlinear in the displacements, due to geometrical effects of the lattice. Whilst it is possible to simplify the equations of motion, by ignoring the onsite potential, and so generate a FPU lattice, the analysis of such systems is more complicated than the Klein-Gordon case, in which the onsite potential is retained. Hence, here we consider the Klein-Gordon system and, in a later paper analyse the 2D FPU system.

\section{Formulation of the 2D lattice equations}

We consider a spring-mass system with square periodic geometry in which particles at the nodes are permitted to move in both directions within the plane of the lattice. Each node has unit mass and is coupled to its four nearest neighbours by nonlinear springs and to its four second (diagonal) neighbours with linear springs. For the sake of generality we include a nonlinear on-site potential in addition to nearest neighbour interactions. Later, we separately consider the Klein-Gordon case, where there is an onsite interaction with linear nearest-neighbour interactions, and the FPU case, which has nonlinear nearest-neighbour interactions but no onsite potential. 

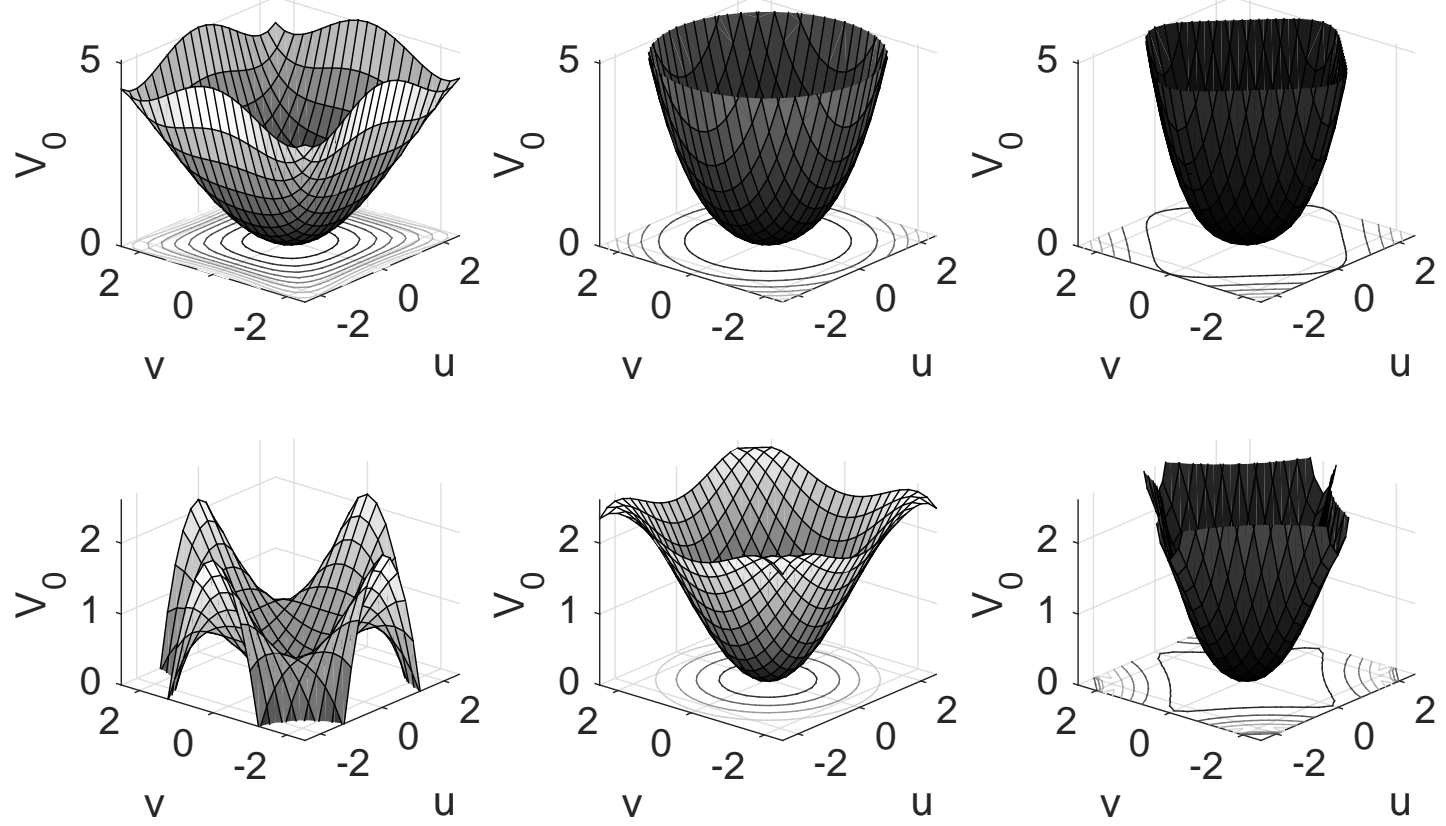

Fig. 1. Illustration of the onsite potential energy function (2.1) for $\omega=1, \lambda=0.2$ (top row) and $\lambda=-0.1$ (bottom row) and $\mu=-0.5$ (left), $\mu=0$ (centre), and $\mu=1$ (right).

At equilibrium, the particles are spaced regularly in the horizontal, and vertical directions and all the springs unextended. Introducing lattice basis vectors $\mathbf{i}=$ $[1,0]^{T}$ and $\mathbf{j}=[0,1]^{T}$, the nodes can be referenced by $(m, n)$, the equilibrium position being $m \mathbf{i}+n \mathbf{j}$. The displacement from equilibrium is then described by the vector $\left(u_{m, n}(t), v_{m, n}(t)\right)$.

To obtain the equations of motion, we first construct the Hamiltonian. The onsite potential is given by

$$
\begin{aligned}
V_{0}\left(u_{m, n}, v_{m, n}\right)= & \frac{1}{2} \Omega^{2} r_{m, n}^{2}+\frac{1}{4} \lambda r_{m, n}^{4}+\frac{1}{2} \mu u_{m, n}^{2} v_{m, n}^{2}, \\
& \text { where } r_{m, n}^{2}=u_{m, n}^{2}+v_{m, n}^{2} .
\end{aligned}
$$

Here, the term involving $\Omega$ is a harmonic potential, whilst the $\lambda$ term gives a nonlinear contribution to the potential which is still isotropic in that it depends purely on the absolute displacement from equilibrium, so contains no directional dependence. The case $\lambda>0$ corresponds to a hardening nonlinearity, and $\lambda<0$ to a softening nonlinearity. The term involving $\mu$ is a nonlinear anisotropic contribution to the onsite potential. In Figure 1 we illustrate the range of forms of the potential $V_{0}(u, v)$ showing the effect of the nonlinearity, $\lambda$, with $\lambda>0$ in the top row, and $\lambda<0$ in the lower row; and the effect of the anisotropy parameter, $\mu$, with $\mu<0$ on the left, $\mu=0$ in the centre, and $\mu>0$ on the right. If we were considering a fully three-dimensional lattice 
system, composed of parallel planes of atoms, the term $V_{0}$ would model the effects of atoms in the planes above and below the plane of interest.

In addition to $V_{0}$, there are potential energies due to the stored energy of the horizontal, vertical and diagonal springs, which we denote by $V_{h}, V_{v}, V_{d}$ and $V_{e}$ respectively. These are given by

$$
V_{h}(\phi)=V_{v}(\phi)=\frac{1}{2} \phi^{2}+\frac{1}{3} a \phi^{3}+\frac{1}{4} b \phi^{4}, \quad V_{d}(\phi)=V_{e}(\phi)=\frac{1}{2} \gamma \phi^{2},
$$

where the extensions of the springs $\phi_{*}$ in the horizontal $\left(\phi_{h}\right)$, vertical $\left(\phi_{v}\right)$ and diagonal $\left(\phi_{d}, \phi_{e}\right)$ directions are given by

$$
\begin{aligned}
& \phi_{m, n, h}=\sqrt{\left(h+u_{m+1, n}-u_{m, n}\right)^{2}+\left(v_{m+1, n}-v_{m, n}\right)^{2}}-h, \\
& \phi_{m, n, v}=\sqrt{\left(u_{m, n+1}-u_{m, n}\right)^{2}+\left(h+v_{m, n+1}-v_{m, n}\right)^{2}}-h, \\
& \phi_{m, n, d}=\sqrt{\left(h+u_{m+1, n+1}-u_{m, n}\right)^{2}+\left(h+v_{m+1, n+1}-v_{m, n}\right)^{2}}-h \sqrt{2}, \\
& \phi_{m, n, e}=\sqrt{\left(h+u_{m+1, n-1}-u_{m, n}\right)^{2}+\left(h-v_{m+1, n-1}+v_{m, n}\right)^{2}}-h \sqrt{2} .
\end{aligned}
$$

The definitions of $u_{m, n}, v_{m, n}, \phi_{m, n, h}, \phi_{m, n, v}, \phi_{m, n, d}$ and $\phi_{m, n, e}$ are illustrated in Figure 2.

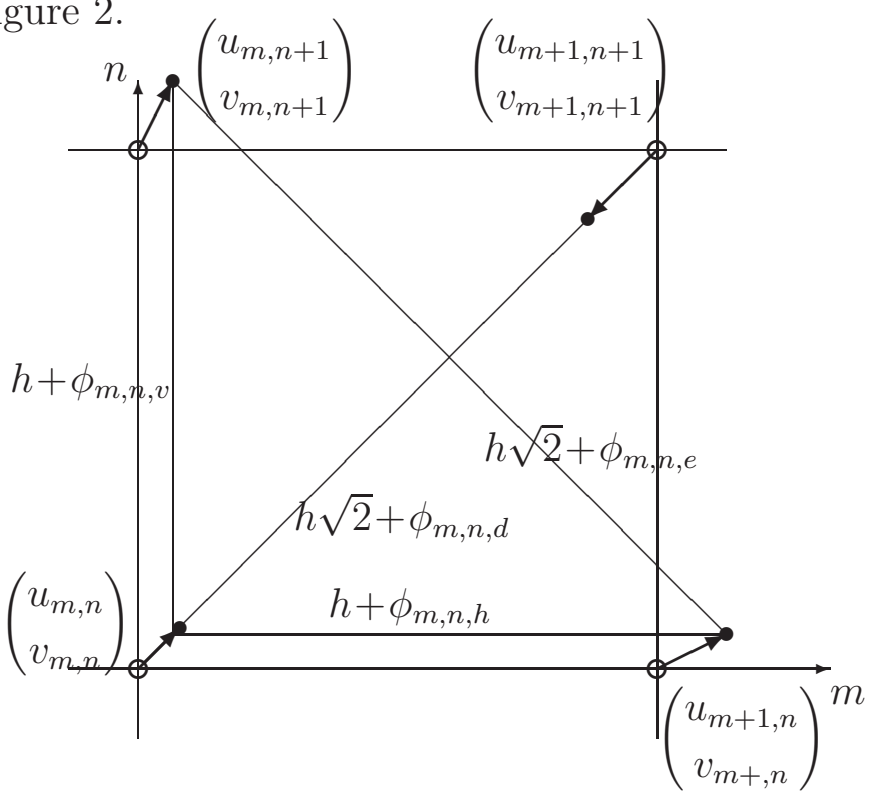

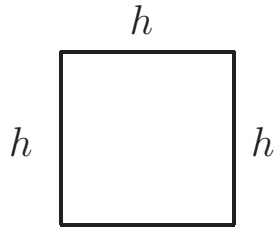

$h$
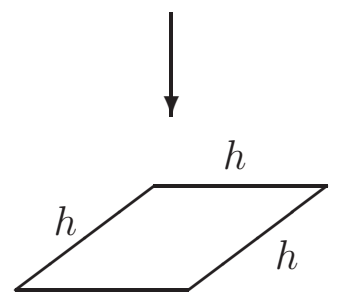

$h$

Fig. 2. Left: Illustration of unit cell of the lattice, with definitions of the displacements $\left(u_{m, n}, v_{m, n}\right)$, from equilibrium positions marked with open circles to the displaces positions, marked with filled circles, and the corresponding spring extensions $\phi_{m, n, h}, \phi_{m, n, v}, \phi_{m, n, d}, \phi_{m, n, e}$ defined by (2.3)-(2.6). Right: Illustration of the shearing of the lattice which causes a degeneracy of the ground state in the special case $V_{0}=0$ with $\gamma=0$, that is, no onsite potential and no diagonal interactions.

The Hamiltonian is given by 


$$
\begin{array}{r}
H=\sum_{m, n}\left\{\frac{1}{2}\left(\frac{\mathrm{d} u_{m, n}}{\mathrm{~d} t}\right)^{2}+\frac{1}{2}\left(\frac{\mathrm{d} v_{m, n}}{\mathrm{~d} t}\right)^{2}+V_{0}\left(u_{m, n}, v_{m, n}\right)+V_{h}\left(\phi_{m, n, h}\right)\right. \\
\left.+V_{v}\left(\phi_{m, n, v}\right)+V_{d}\left(\phi_{m, n, d}\right)+V_{e}\left(\phi_{m, n, e}\right)\right\}
\end{array}
$$

where the spring extensions $\phi_{m, n, *}$ are defined in (2.3) -(2.6). We obtain the equations of motion by applying Hamilton's principle to (2.7). To simplify the following calculations, we introduce the differences

$$
\begin{aligned}
\delta_{+0} u & =\left(u_{m+1, n}-u_{m, n}\right), \quad \delta_{0+} u=\left(u_{m, n+1}-u_{m, n}\right), \\
\delta_{++} u & =\left(u_{m+1, n+1}-u_{m, n}\right), \quad \delta_{+-} u=\left(u_{m+1, n-1}-u_{m, n}\right),
\end{aligned}
$$

with similar definitions made for $\delta_{* *} v($ where $*= \pm)$. Since we are interested in small amplitude disturbances, we expand all quantities in (2.3)-(2.6) assuming $u, v \ll h$, obtaining

$$
\begin{aligned}
& \phi_{m, n, h}=\delta_{+0} u+\frac{\left(\delta_{+0} v\right)^{2}}{2 h}-\frac{\left(\delta_{+0} u\right)\left(\delta_{+0} v\right)^{2}}{2 h^{2}}, \\
& \phi_{m, n, v}=\delta_{0+} v+\frac{\left(\delta_{0+} u\right)^{2}}{2 h}-\frac{\left(\delta_{0+} v\right)\left(\delta_{0+} u\right)^{2}}{2 h^{2}}, \\
& \phi_{m, n, d}=\frac{\left(\delta_{++} u+\delta_{++} v\right)}{\sqrt{2}}+\frac{\left(\delta_{++} u-\delta_{++} v\right)^{2}}{4 h \sqrt{2}}-\frac{\left(\delta_{++} u+\delta_{++} v\right)\left(\delta_{++} u-\delta_{++} v\right)^{2}}{8 h^{2} \sqrt{2}}, \\
& \phi_{m, n, e}=\frac{(2.10)}{\sqrt{2}}+\frac{\left(\delta_{+-} u+\delta_{+-} v\right)^{2}}{4 h \sqrt{2}}-\frac{\left(\delta_{+-} u-\delta_{+-} v\right)\left(\delta_{+-} u+\delta_{+-} v\right)^{2}}{8 h^{2} \sqrt{2}} .
\end{aligned}
$$

Thus, to quartic terms in $u, v$, the Hamiltonian (2.7) can be written

$$
\begin{aligned}
& H=\sum_{m, n} \frac{1}{2}\left(\frac{\mathrm{d} u_{m, n}}{\mathrm{~d} t}\right)^{2}+\frac{1}{2}\left(\frac{\mathrm{d} v_{m, n}}{\mathrm{~d} t}\right)^{2}+V_{0}+V_{h}+V_{v}+V_{d}+V_{e} \\
& V_{0}=\frac{1}{2} \Omega^{2}\left(u_{m, n}^{2}+v_{m, n}^{2}\right)+\frac{1}{4} \lambda\left(u_{m, n}^{2}+v_{m, n}^{2}\right)^{2}+\frac{1}{2} \mu u_{m, n}^{2} v_{m, n}^{2}
\end{aligned}
$$

where expressions for the quantities $V_{h}, V_{v}, V_{d}$ and $V_{d}$ are given by (2.2), (2.9)-(2.12), with asymptotic expansions quoted in the appendix, see equations (A.1)-(A.4).

Applying Hamilton's equations, the equations of motion are found to be 


$$
\begin{aligned}
& \frac{\mathrm{d}^{2} u_{m, n}}{\mathrm{~d} t^{2}}=-\Omega^{2} u_{m, n}-\lambda u_{m, n}\left(u_{m, n}^{2}+v_{m, n}^{2}\right)-\mu u_{m, n} v_{m, n}^{2}+F_{h}+F_{v}+F_{d}+F_{e}, \\
& \frac{\mathrm{d}^{2} v_{m, n}}{\mathrm{~d} t^{2}}=-\Omega^{2} v_{m, n}-\lambda v_{m, n}\left(u_{m, n}^{2}+v_{m, n}^{2}\right)-\mu u_{m, n}^{2} v_{m, n}+G_{h}+G_{v}+G_{d}+G_{e},
\end{aligned}
$$

where $F_{*}=-\partial V_{*} / \partial u_{m, n}$ and $G_{*}=-\partial V_{*} / \partial v_{m, n}(*=h, v, d, e)$ are the forces in the horizontal, vertical and two diagonal directions, with asymptotic expressions given by (A.5)-(A.12).

\subsection{Complications of the $2 D$ system}

Let us consider generalising the simplification of the 1D lattice to the KleinGordon equation (1.3) to the two-dimensional setting. If we attempt to simplify the system by setting $a=b=0$ in (2.2) to remove the nonlinear nearestneighbour interactions, little simplification is gained since geometric nonlinearities are generated from the two-dimensionality of the problem, through additional terms, such as those appearing in (A.5)-(A.12), for example, the diagonal interactions $(\gamma)$ and terms where a vertical displacement generates a horizontal force and vice versa. Thus in two-dimensions, we may as well consider nonlinear nearest-neighbour interactions (that is, $a \neq 0 \neq b$ ).

Another simplification we could consider is putting $\lambda=\mu=\Omega=0$, which is equivalent to assuming $V_{0}=0$ and retaining $\gamma>0$ simplifies $2 \mathrm{D}$ system (2.15)-(2.16) by reducing the general system (2.15)-(2.16) to the FPU form, as in the $1 \mathrm{D}$ case discussed in $\S 1.1$. However, there is no way of defining $\phi$ as a difference of $u_{m, n}, v_{m, n}$ variables to simplify the $2 \mathrm{D}$ equation to something analogous to (1.5); we would need four such variables $\left(\phi_{m, n}=u_{m+1, n}-u_{m, n}\right.$, $\left.\widetilde{\phi}_{m, n}=u_{m, n+1}-u_{m, n}, \psi_{m, n}=v_{m+1, n}-v_{m, n}, \widetilde{\psi}_{m, n}=v_{m, n+1}-v_{m, n}\right)$. Instead, we have to analyse the more complicated equations (2.15)-(2.16) directly.

A final simplification that we comment on is the case of vanishing diagonal interactions $(\gamma=0)$ in the FPU case $V_{0}=0$. Here, the ground state becomes degenerate. If we describe the absolute positions of each node in the square lattice at equilibrium by $\left(U_{m, n}, V_{m, n}\right)=(m h, n h)$; then we can construct a sheared lattice in which all horizontal and vertical springs still have length $h$; namely we define the absolute nodal positions by

$$
\left(\begin{array}{c}
\widehat{U}_{m, n} \\
\widehat{V}_{m, n}
\end{array}\right)=\left(\begin{array}{c}
m h+n h \cos \theta \\
n h \sin \theta
\end{array}\right) .
$$

This corresponds to displacements from equilibrium given by $\left(u_{m, n}, v_{m, n}\right)=$ 
$\left(\widehat{U}_{m, n}-U_{m, n}, \widehat{V}_{m, n}-V_{m, n}\right)=(n h \cos \theta,-n h(1-\sin \theta))$. Thus instead of being square, the lattice cell is deformed into a rhombus, with diagonals of lengths $d_{\text {short }}=2 h \sin \frac{1}{2} \theta$ and $d_{\text {long }}=2 h \cos \frac{1}{2} \theta$. This is illustrated in the right panel of Figure 2. Reintroducing an energy due to diagonal interactions, given by (2.2) and $V_{\text {diag }}=V_{d}$, we find

$$
V_{\text {diag }}=\sum \frac{1}{2} \gamma(d-\sqrt{2} h)^{2}=8 \gamma h^{2} \sin ^{2}\left(\frac{1}{4} \theta-\frac{1}{8} \pi\right)
$$

which is minimised at $\theta=\frac{1}{2} \pi$. Thus, in the FPU case, a nonzero $\gamma$ term is necessary to remove the degeneracy of the ground state solution, and stabilises the lattice's square geometry.

Thus, in the remainder of this paper we consider the $2 \mathrm{D} \mathrm{KG}$ lattice, that is, $V_{0} \neq 0$, in the general case where $\gamma>0, \Omega>0$ and $a \neq 0 \neq b$. We comment on the special cases where $\Omega$ and $\gamma$ become vanishingly small, although detailed analysis of the FPU case will be considered in a later paper.

\section{Asymptotic solution}

We now seek an approximate analytic solution to the equations (2.15) and (2.16) by applying the method of multiple scales, which is covered in advanced texts such as Bender \& Orszag [4] and Murray [25]. We define a small parameter, $\epsilon$ as the amplitude of the wave, and introduce scaled variables

$$
x=\epsilon m, \quad y=\epsilon n, \quad \tau=\epsilon t, \quad T=\epsilon^{2} t,
$$

to describe evolution over the large space scales, and long and very long timescales. We retain the $\mathcal{O}(1)$ variables $m, n, t$, introduce the phase $\psi=$ $k m+l n+\omega t$ and seek solutions of the form

$$
\begin{aligned}
u_{m, n}(t)= & \epsilon e^{i \psi} F+\epsilon^{2}\left(G_{0}+e^{i \psi} G_{1}+e^{2 i \psi} G_{2}\right)+\epsilon^{3}\left(H_{0}+e^{i \psi} H_{1}+e^{2 i \psi} H_{2}+e^{3 i \psi} H_{3}\right) \\
& +\ldots+c . c . \\
v_{m, n}(t)= & \epsilon e^{i \psi} P+\epsilon^{2}\left(Q_{0}+e^{i \psi} Q_{1}+e^{2 i \psi} Q_{2}\right)+\epsilon^{3}\left(R_{0}+e^{i \psi} R_{1}+e^{2 i \psi} R_{2}+e^{3 i \psi} R_{3}\right) \\
& +\ldots+\text { c.c. }
\end{aligned}
$$

Here, $F, G_{j}, H_{j}, P, Q_{j}$ and $R_{j}(j=0,1,2,3$,$) are all functions of the slow$ variables $x, y, \tau, T$ (3.1). We substitute the ansatz (3.2) and (3.3), into the relevant motion equations (2.15) and (2.16), equate the coefficients of each frequency $e^{i j \psi}$ at each order of $\epsilon$ to find two sets of equations, which we analyse in order below. 


\subsection{The dispersion relation $-\mathcal{O}\left(\epsilon e^{i \psi}\right)$ terms}

From the $\mathcal{O}\left(\epsilon \mathrm{e}^{i \psi}\right)$ terms of equation (2.15)-(2.16), which give two equations relating $F$ and $P$ we obtain

$$
\mathbf{M}\left(\begin{array}{l}
F \\
P
\end{array}\right):=\left(\begin{array}{cc}
S-2 \cos k-\omega^{2} & 2 \gamma(1-\cos k \cos l) \\
2 \gamma(1-\cos k \cos l) & S-2 \cos l-\omega^{2}
\end{array}\right)\left(\begin{array}{l}
F \\
P
\end{array}\right)=\mathbf{0}
$$

where

$$
S=\Omega^{2}+2+2 \gamma(1-\cos k \cos l)
$$

We are interested in solutions where $(F, P)^{T} \neq 0$, thus (3.4) is in effect an eigenvalue problem, with the eigenvalue being $\omega^{2}$. We require the determinant of the matrix to be zero, which gives the dispersion relation

$$
\begin{aligned}
\omega^{2}(k, l) & =S-\cos k-\cos l \pm \sqrt{D} \\
D & =(\cos k-\cos l)^{2}+4 \gamma^{2}(1-\cos k \cos l)^{2} .
\end{aligned}
$$

This dispersion relation describes the dependence of the temporal frequency of the wave $(\omega)$ on the wavenumbers $(k, l)$. For small amplitude solutions to be stable, we require $\omega \in \mathbb{R}$ which is equivalent to $\omega^{2} \geq 0$. Consideration of the dispersion relation for $l=0$ and small $k$ then implies $\gamma \geq 0$, thus we impose this condition in the following.

The negative square root in (3.7) leads to an acoustic branch or surface in $(k, l, \omega)$ space of lower frequencies, which we denote by $\omega_{a c}$, and the positive root in (3.7) we donate by $\omega_{\text {opt }}$, which we describe as the optical branch. Note that the two surfaces overlap in frequencies, and there is no gap between the two, that is we do not have $\omega_{a c}<\omega_{\text {gap }}<\omega_{\text {opt }}$. In following expressions, the upper sign corresponds to the optical branch, and the lower sign to the acoustic branch.

The surfaces are illustrated in Figure 3 for a variety of choices of $\gamma, \Omega$. In all cases, the surfaces are periodic in both $k$ and $l$, with period $2 \pi$ in each direction. The surfaces meet, and have minima at $k=l=0$, the classification of the maxima and saddle points is more complicated, and is summarised in Table 1. When $\gamma=0$, the two modes decouple into $\omega^{2}=\Omega^{2}+4 \sin ^{2}\left(\frac{1}{2} k\right)$ and $\omega^{2}=\Omega^{2}+4 \sin ^{2}\left(\frac{1}{2} l\right)$.

It is not sufficient to obtain the eigenvalue, $\omega$, we also require the form of the solution, given by $F, P$, hence we need the eigenvector of (3.4) as well. We write this as $(F, P)^{T}=F(1, C)^{T}$, and plot the constant $C(k, l)$ in figure 4 . Solving (3.4) we obtain $C(k, l)$ as 

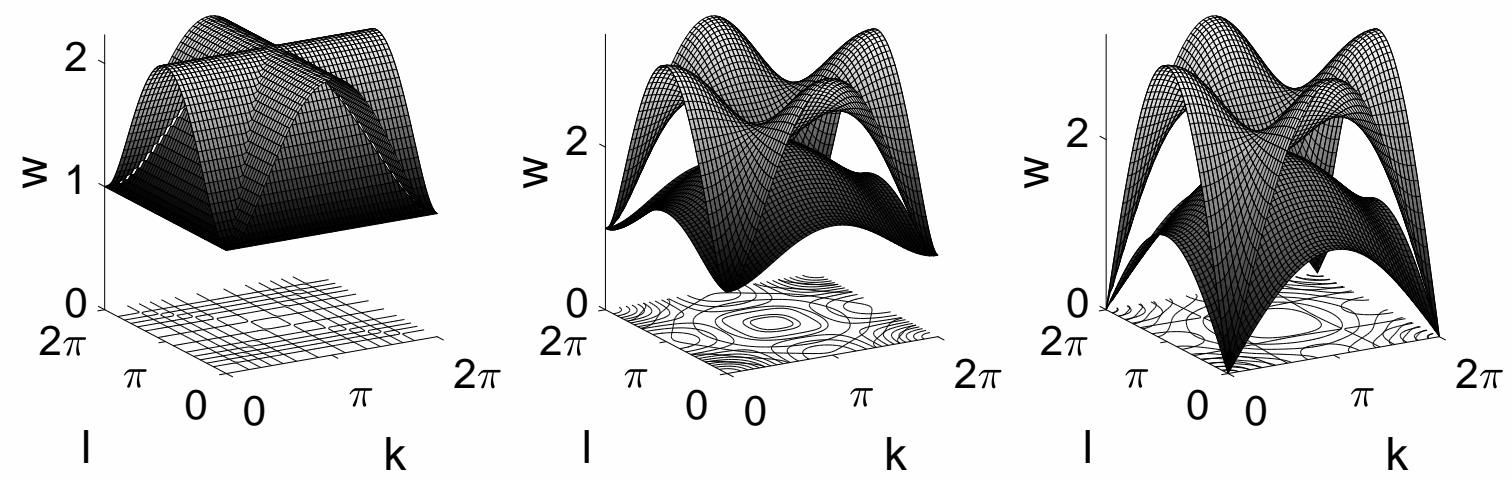

Fig. 3. Plots of the dispersion relation (3.7). Left panel: $\Omega=1, \gamma=0$, the KG case with no diagonal interactions; centre: $\Omega=1, \gamma=1$, the KG case with diagonal interactions; right panel: $\Omega=0, \gamma=1$ ), the FPU case, where there is no onsite
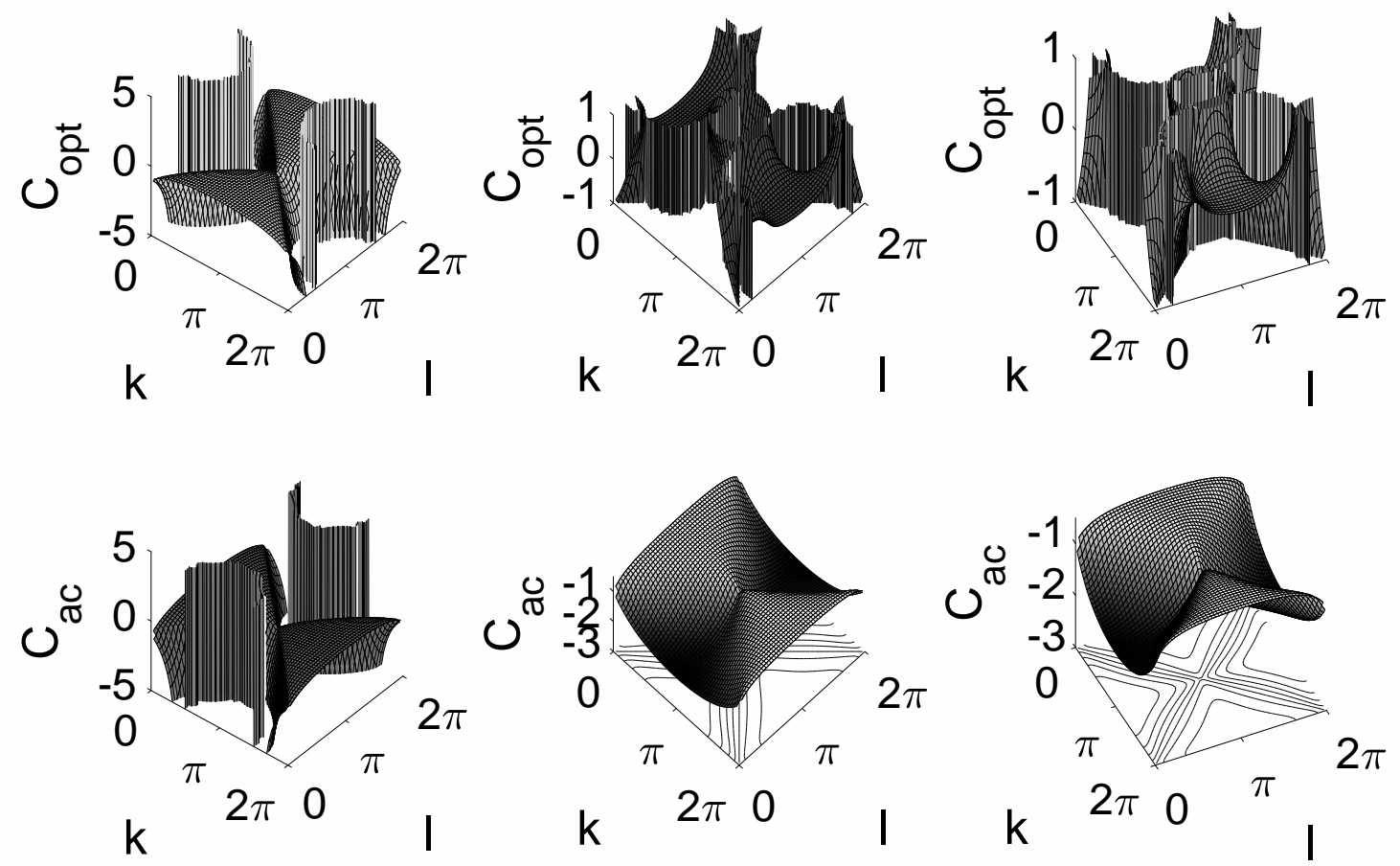

Fig. 4. Plots of the constant $C=P / F$ given by (3.8)-(3.9) with parameters as in Figure 3, namely: left panels: $\Omega=1, \gamma=0$, the KG case with no diagonal interactions; centre panels: $\Omega=1, \gamma=1$, the KG case with diagonal interactions; right panels: $\Omega=0, \gamma=1$, the FPU case where the onsite potential vanishes. In each case the upper plot shows the optical case, and the lower plot the acoustic case.

$$
\begin{aligned}
C_{o p t}(k, l) & =\frac{\cos k-\cos l-2 \gamma(1-\cos k \cos l)+\sqrt{D}}{\cos k-\cos l+2 \gamma(1-\cos k \cos l)-\sqrt{D}} \\
C_{a c}(k, l) & =\frac{\cos k-\cos l-2 \gamma(1-\cos k \cos l)-\sqrt{D}}{\cos k-\cos l+2 \gamma(1-\cos k \cos l)+\sqrt{D}}
\end{aligned}
$$




\begin{tabular}{|c|c|c|}
\hline Location & $\begin{array}{c}\text { Type of critical point } \\
\text { on acoustic surface, } \omega_{a c}\end{array}$ & $\begin{array}{c}\text { Type of critical point } \\
\text { on optical surface, } \omega_{\text {opt }}\end{array}$ \\
\hline \hline $\begin{array}{c}(k, l)=(0,0) \\
- \text { surfaces meet }\end{array}$ & $\begin{array}{c}\text { Global minimum: } \\
\omega_{a c}=\Omega\end{array}$ & $\begin{array}{c}\text { Global minimum: } \\
\omega_{\text {opt }}=\Omega\end{array}$ \\
\hline $\begin{array}{c}(k, l)=(\pi, 0) \\
\text { or }(k, l)=(0, \pi)\end{array}$ & $\begin{array}{c}\text { Saddle point: } \omega_{a c}= \\
\Omega^{2}+2+4 \gamma-2 \sqrt{1+4 \gamma^{2}}\end{array}$ & $\begin{array}{c}\text { Global maximum: } \omega_{\text {opt }}= \\
\Omega^{2}+2+4 \gamma+2 \sqrt{1+4 \gamma^{2}}\end{array}$ \\
\hline $\begin{array}{c}\text { For } 0<\gamma<\frac{1}{4}: \\
(k, l)=(\pi, \pi)\end{array}$ & Global maximum: & Degenerate saddle: \\
- surfaces meet & $\omega_{a c}=\sqrt{\Omega^{2}+4}$ & $\omega_{\text {opt }}=\sqrt{\Omega^{2}+4}$ \\
\hline For $\gamma>\frac{1}{4}:$ & Global maximum: & Local minimum: \\
$(k, l)=(\pi, \pi)$ & $\omega_{a c}=\sqrt{\Omega^{2}+4}$ & $\omega_{\text {opt }}=\sqrt{\Omega^{2}+4}$ \\
- surfaces meet & & Saddle point: $\omega_{\text {opt }}=$ \\
\hline For $\gamma>\frac{1}{4}:$ & $\mathrm{N} / \mathrm{A}$ & $\sqrt{\Omega^{2}+2+4 \gamma-(1 / 4 \gamma)}$ \\
$k_{*}=\cos ^{-1} \frac{-1}{4 \gamma}$ & & \\
$(k, l)=\left(k_{*}, k_{*}\right)$ & & \\
$\left(k_{*}, 2 \pi-k_{*}\right),\left(2 \pi-k_{*}, k *\right)$ & & \\
and $\left(2 \pi-k_{*}, 2 \pi-k_{*}\right)$ & & \\
\hline
\end{tabular}

Table 1

Summary of the locations of stationary points of the dispersion surfaces in wavenumber-space $(k, l)$, from $(3.7)$. At $(k, l)=(0,0),(\pi, 0),(0, \pi)$ and $(\pi, \pi)$ both optical and acoustic branches have stationary points. The type of critical point at $(\pi, \pi)$ depends on the sign of $\gamma-\frac{1}{4}$. In the case of $\gamma>\frac{1}{4}$, there is in additional critical point in the optical branch of the dispersion relation.

Note that these results imply both that $C_{a c}, C_{o p t} \in \mathbb{R}$ and $C_{a c} C_{o p t}=-1$.

\subsection{Special cases}

If we were to consider the simplest possible $\mathrm{KG}$ case, where there are no diagonal springs (that is, $\gamma=0$ ), so that only the onsite potential removes the degeneracy of the solution (2.17), then the leading order problem (3.4) still becomes degenerate.

The case $\gamma=0$ is an oversimplification of the system, since then at leading order, (3.7) results in $\omega^{2}=\Omega^{2}+2-2 \cos k$ and $\omega^{2}=\Omega^{2}+2-2 \cos l$. The resulting eigenvectors are $(F, P)=(F, 0)$ and $(F, P)=(0, P)$ respectively, 
corresponding to $C=0$ or $C=\infty$, which indicates that solutions involve motion in only one direction. That is, either $v_{m, n}=0$ or $u_{m, n}=0$ for all $m, n$. Such solutions are not genuinely two-dimensional modes. Whilst such solutions may formally exist, the ansatz (3.2)-(3.3) does not generate an envelope which is localised in both spatial directions. In the case $\gamma=0$, there may be solutions with different scalings in the $m, n$ directions with other asymptotic magnitudes for $u_{m, n}$ and $v_{m, n}$. However, we do not consider the case $\gamma=0$ any further in this paper, instead we analyse the case $\gamma>0$ where $C \neq 0, \infty$. The expressions for $C_{a c}, C_{o p t}$ will be used later, in the determination of expressions for the functions $G_{0}, G_{1}, G_{2}, Q_{0}, Q_{1}$ and $Q_{2}$, in terms of $F$. We retain the nonlinear onsite potential, taking $\Omega>0$ and $\lambda \neq 0$ which means that $u_{m, n}=0=v_{m, n}$ is the unique stable equilibrium configuration. We also retain general values for $a, b$, since setting $a=b=0$ does not lead to any significant simplification, since the presence of $\gamma>0$ generates similar nonlinearities.

\subsection{Zeroth harmonic $-\mathcal{O}\left(\epsilon^{2} e^{0}\right)$}

From the $\mathcal{O}\left(\epsilon^{2} e^{0 i \psi}\right)$ terms in $(2.15)-(2.16)$, we obtain

$$
0=-\Omega^{2}\left(G_{0}+G_{0}^{*}\right), \quad 0=-\Omega^{2}\left(Q_{0}+Q_{0}^{*}\right),
$$

Due to the form of the ansatz (3.2)-(3.3), the imaginary parts of $G_{0}$ and $Q_{0}$, $\operatorname{Im}\left(G_{0}\right)$ and $\operatorname{Im}\left(Q_{0}\right)$ do not contribute to $u_{m, n}$ or $v_{m, n}$, Thus, without loss of generality we assume $\operatorname{Im}\left(G_{0}\right)=0=\operatorname{Im}\left(Q_{0}\right)$ and so $G_{0}=G_{0}^{*}$ and $Q_{0}=Q_{0}^{*}$. Equation (3.10) implies that the real parts of $G_{0}, Q_{0}$ are also zero, and so $G_{0}=Q_{0}=0$.

\subsection{The second harmonic $-\mathcal{O}\left(\epsilon^{2} e^{2 i \psi}\right)$ terms}

Considering the terms at $\mathcal{O}\left(\epsilon^{2} \mathrm{e}^{2 i \psi}\right)$, we obtain two equations which relate the amplitude of the second harmonic terms $G_{2}, Q_{2}$ to the nonlinear terms $F^{2}$, $P^{2}=C^{2} F^{2}$ and $F P=C F^{2}$, namely

$$
\begin{aligned}
& {\left[\begin{array}{l}
\left.\Omega^{2}-4 \omega^{2}+4 \sin ^{2} k+2 \gamma \sin ^{2}(k+l)+2 \gamma \sin ^{2}(k-l)\right] G_{2} \\
+ \\
\quad+2 \gamma\left[\sin ^{2}(k+l)+\sin ^{2}(k-l)\right] Q_{2}=F^{2} r_{1},
\end{array}\right.} \\
& 2 \gamma\left[\sin ^{2}(k+l)+\sin ^{2}(k-l)\right] G_{2} \\
& \quad+\left[\Omega^{2}-4 \omega^{2}+4 \sin ^{2} l+2 \gamma \sin ^{2}(k+l)+2 \gamma \sin ^{2}(k-l)\right] Q_{2}=F^{2} r_{2},
\end{aligned}
$$

where 


$$
\begin{aligned}
r_{1}= & {\left[-16 a i \sin ^{3} \frac{1}{2} k \cos \frac{1}{2} k-\frac{6 \gamma i}{h} \sin ^{3} \frac{k+l}{2} \cos \frac{k+l}{2}-\frac{6 \gamma i}{h} \sin ^{3} \frac{k-l}{2} \cos \frac{k-l}{2}\right] } \\
& +\left[\frac{4 \gamma i}{h} \sin ^{3} \frac{k+l}{2} \cos \frac{k+l}{2}-\frac{4 \gamma i}{h} \sin ^{3} \frac{k-l}{2} \cos \frac{k-l}{2}-\frac{16 i}{h} \sin ^{3} \frac{1}{2} l \cos \frac{1}{2} l\right] C \\
& +\left[-\frac{8 i}{h} \sin ^{3} \frac{1}{2} k \cos \frac{1}{2} k+\frac{2 \gamma i}{h} \sin ^{3} \frac{k+l}{2} \cos \frac{k+l}{2}-\frac{2 \gamma i}{h} \sin ^{3} \frac{k-l}{2} \cos \frac{k-l}{2}\right] C^{2}, \\
r_{2}= & {\left[+\frac{2 \gamma i}{h} \sin ^{3} \frac{k+l}{2} \cos \frac{k+l}{2}-\frac{2 \gamma i}{h} \sin ^{3} \frac{l-k}{2} \cos \frac{l-k}{2}-\frac{8 i}{h} \sin ^{3} \frac{1}{2} l \cos \frac{1}{2} l\right] } \\
& +\left[\frac{4 \gamma i}{h} \sin ^{3} \frac{k+l}{2} \cos \frac{k+l}{2}-\frac{4 \gamma i}{h} \sin ^{3} \frac{l-k}{2} \cos \frac{l-k}{2}-\frac{16 i}{h} \sin ^{3} \frac{1}{2} k \cos \frac{1}{2} k\right] C \\
& +\left[-16 a i \sin ^{3} \frac{1}{2} l \cos \frac{1}{2} l-\frac{6 \gamma i}{h} \sin ^{3} \frac{k+l}{2} \cos \frac{k+l}{2}-\frac{6 \gamma i}{h} \sin ^{3} \frac{l-k}{2} \cos \frac{l-k}{2}\right] C^{2} .
\end{aligned}
$$

Although lengthy, this is a straightforward system of two linear equations for $G_{2}, Q_{2}$ of the form $\mathbf{Z}\left(\begin{array}{l}G_{2} \\ Q_{2}\end{array}\right)=F^{2}\left(\begin{array}{l}r_{1} \\ r_{2}\end{array}\right)$, which can be solved by inverting the symmetric $2 \times 2$ matrix $\mathbf{Z}$

$$
\mathbf{Z}=\left(\begin{array}{ll}
Z_{11} & Z_{12} \\
Z_{12} & Z_{22}
\end{array}\right), \quad \text { where } \quad \begin{aligned}
& Z_{12}=2 \gamma\left(\sin ^{2}(k+l)+\sin ^{2}(k-l)\right), \\
& Z_{11}=\Omega^{2}-4 \omega^{2}+4 \sin ^{2} k+Z_{12}, \\
& Z_{22}=\Omega^{2}-4 \omega^{2}+4 \sin ^{2} l+Z_{12},
\end{aligned}
$$

to give

$$
G_{2}=\frac{\left(Z_{22} r_{1}-Z_{12} r_{2}\right) F^{2}}{Z_{11} Z_{22}-Z_{12}^{2}}=\gamma_{G} F^{2}, \quad Q_{2}=\frac{\left(Z_{11} r_{2}-Z_{12} r_{1}\right) F^{2}}{Z_{11} Z_{22}-Z_{12}^{2}}=\gamma_{Q} F^{2}
$$

where $\gamma_{G}, \gamma_{Q}$ are functions of $(k, l)$ which determine the strength of the second harmonic relative to the first harmonic. As shown in Figure 5, these functions are well-defined almost everywhere in $(k, l)$-space.

\subsection{First consistency condition and the velocity $-\mathcal{O}\left(\epsilon^{2} e^{i \psi}\right)$ terms}

Following our asymptotic strategy of substituting the ansatz (3.2)-(3.3) into the governing equations (2.15)-(2.16) and equating terms of $\mathcal{O}\left(\epsilon^{2} \mathrm{e}^{i \psi}\right)$ we find

$$
\mathbf{M}\left(\begin{array}{l}
G_{1} \\
Q_{1}
\end{array}\right)=\left(\begin{array}{l}
A_{2} \\
B_{2}
\end{array}\right)
$$

where the matrix $\mathbf{M}$ is as given in (3.4), and the RHS vector is given by 

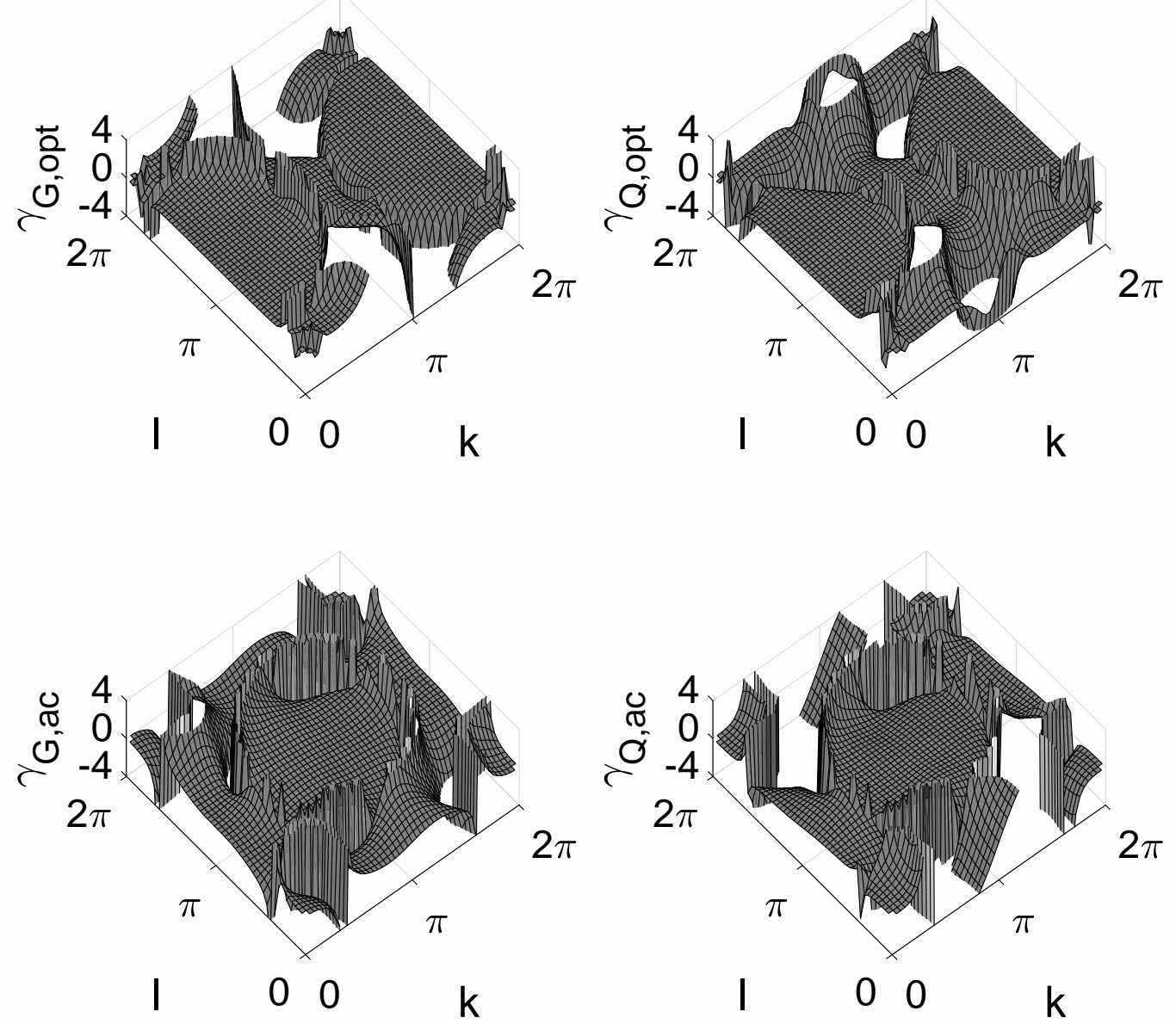

Fig. 5. Plots of the functions $\gamma_{G}$ (left panels), and $\gamma_{Q}$ (right) against $(k, l)$ as defined in (3.16) for the case $\Omega=1$ and $\gamma=1$; upper panels illustrate the optical mode; and lower panels, the acoustic mode.

$$
\begin{aligned}
A_{2}= & 2 i \omega F_{\tau}+2 i \sin k F_{x}+i \gamma\left(F_{x}+F_{y}+P_{x}+P_{y}\right) \sin (k+l) \\
& +i \gamma\left(F_{x}-F_{y}+P_{x}-P_{y}\right) \sin (k-l), \\
B_{2}= & 2 i \omega P_{\tau}+2 i \sin l P_{y}+i \gamma\left(F_{x}+F_{y}+P_{x}+P_{y}\right) \sin (k+l) \\
& +i \gamma\left(F_{x}-F_{y}+P_{x}-P_{y}\right) \sin (k-l) .
\end{aligned}
$$

Equation (3.19) can be derived from (3.18) by the transformations $k \leftrightarrow l$, $F \leftrightarrow P, x \leftrightarrow y$.

The system of equations (3.17) for $G_{1}, Q_{1}$ is quite different to the system in the previous section, since here, the matrix $\mathbf{M}$ is singular, and so not invertible. The system (3.17) only has solutions if a consistency condition is met. This requirement is the Fredholm alternative, which can be expressed as: the system of equations (3.17)-(3.19) only has a solution if the RHS vector, that is, $\left(A_{2}, B_{2}\right)^{T}$, is in the range of the matrix $M$. If this condition is satisfied, the 
system then has a one-parameter family of solutions of the form

$$
\left(\begin{array}{l}
G_{1} \\
Q_{1}
\end{array}\right)=\widehat{G}\left(\begin{array}{l}
1 \\
C
\end{array}\right)+\widetilde{G}\left(\begin{array}{c}
C \\
-1
\end{array}\right),
$$

where $\widetilde{G}$ is determined by the equations (3.17)-(3.19), and $\widehat{G}$ is arbitrary. The vector $(C,-1)^{T}$ is chosen as it is clearly not parallel to $(1, C)$ : this choice is made to simplify later calculations. Here, we take $\widehat{G}=0$ by absorbing such a component into the leading order term $F$ (through $F+\epsilon \widehat{G} \mapsto F$ ).

The range of the matrix $\mathbf{M}$ (3.4) can be written as either column of the matrix, or, for simplicity, we take the sum of the columns, rescale, and after some simplification, we can write the range as any vector parallel to $(C,-1)^{T}$. For the RHS vector $\left(A_{2}, B_{2}\right)$ to be parallel to $(C,-1)$, it must be perpendicular to $(1, C)$, thus the consistency condition for a solution of (3.17)-(3.19) to exist is $A_{2}+B_{2} C=0$.

An alternative derivation of the Fredholm condition uses the fact that $\mathbf{M}^{T}$ is singular, hence has a vector $\mathbf{h}$ such that $\mathbf{M}^{T} \mathbf{h}=\mathbf{0}$. To obtain the consistency condition for $\mathbf{M g}=\mathbf{a}$, we consider the expression $\mathbf{h}^{T} \mathbf{M g}=\mathbf{h}$.a. However, we also have $\left(\mathbf{h}^{T} \mathbf{M g}\right)^{T}=\mathbf{g}^{T} \mathbf{M}^{T} \mathbf{h}=\mathbf{g} . \mathbf{0}=0$. Hence we require $\mathbf{h} . \mathbf{a}=0$. Here $\mathbf{h}$ is the normal to the range of $\mathbf{M}$, which in our case is $(1, C)^{T}$.

Since all the terms on the RHS of (3.17) are linear first derivative terms in $F, P$, we seek a travelling wave solution of the form

$$
\begin{array}{ll}
F(x, y, \tau, T)=F(Z, W, T), & Z=x-U \tau \\
P(x, y, \tau, T)=P(Z, W, T), & W=y-V \tau
\end{array}
$$

where the new variables $Z, W$ are travelling wave coordinates in the horizontal and vertical directions. We eliminate $P$ by using $P=C F$, to simplify $A_{2}, B_{2}$ to

$$
\begin{aligned}
A_{2}= & i F_{Z}[-2 \omega U+2 \sin k+2 \gamma(1+C) \sin k \cos l] \\
& +i F_{W}[-2 \omega V+2 \gamma(1+C) \sin l \cos k], \\
B_{2}= & i F_{Z}[-2 \omega U C+2 \gamma(1+C) \sin k \cos l] \\
& +i F_{W}[-2 \omega V C+2 C \sin l+2 \gamma(1+C) \sin l \cos k],
\end{aligned}
$$

and then use the consistency condition $A_{2}+B_{2} C=0$ to determine the velocities $U=U(k, l), V=V(k, l)$ as 


$$
\begin{aligned}
& U=\frac{\sin k}{\omega\left(1+C^{2}\right)}\left(1+\gamma(1+C)^{2} \cos l\right), \\
& V=\frac{\sin l}{\omega\left(1+C^{2}\right)}\left(C^{2}+\gamma(1+C)^{2} \cos k\right) .
\end{aligned}
$$

These are illustrated in Figure 6 in the case $\Omega=1, \gamma=1$. Due to the symmetry of the system under the transformation $k \leftrightarrow l, F \leftrightarrow P, x \leftrightarrow y$, the velocities
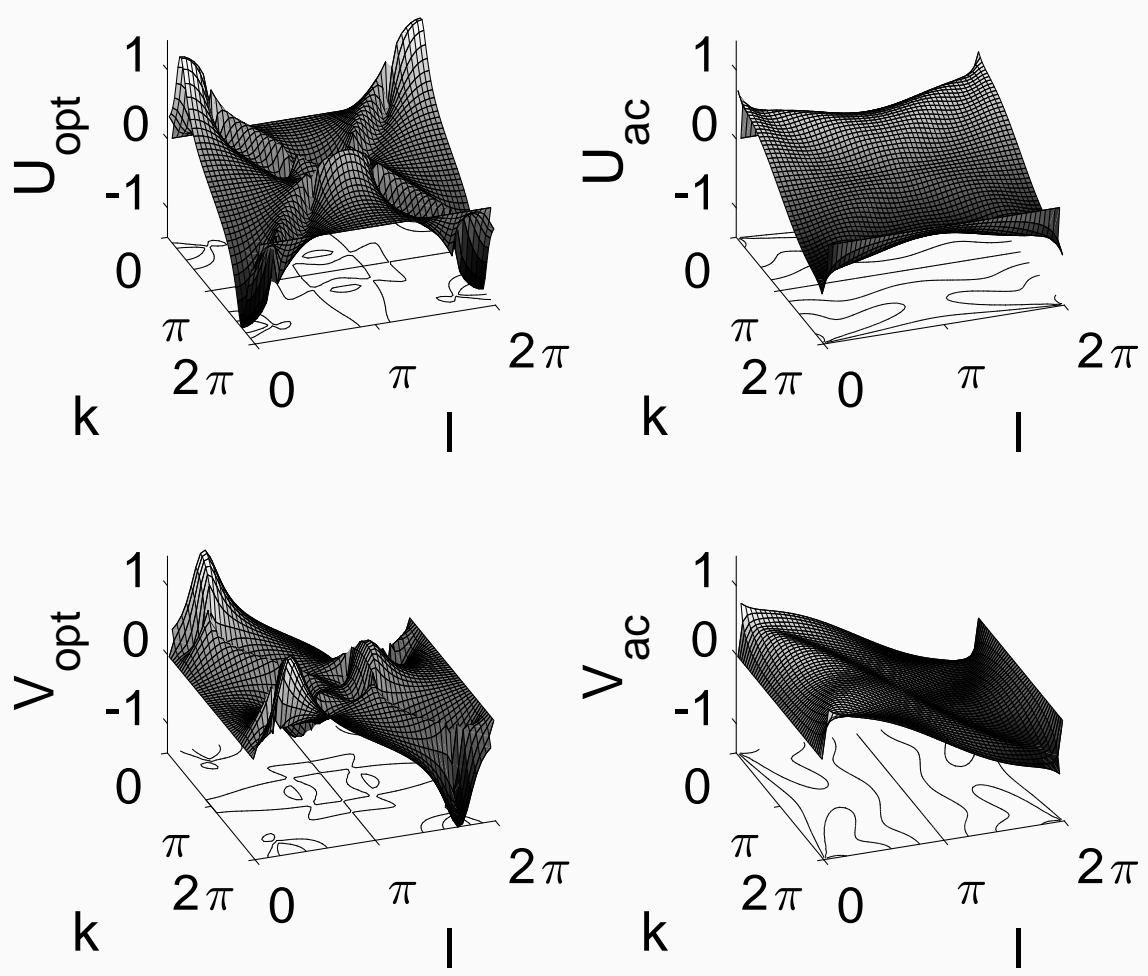

Fig. 6. Plots of the velocities (3.24)-(3.25) with $\Omega=1, \gamma=1$; left panels show the results for the optical mode; and the right panels illustrate the acoustic mode; horizontal velocities $U$ on the top row, vertical velocities, $V$, on the bottom row.

The Fredholm alternative $A_{2}+B_{2} C=0$ which results in (3.21)-(3.25) only provides a partial solution to (3.17)-(3.19), in that it means that the two equations are multiples of each other. Thus if one is solved, the other is too, however, we still need to provide a solution to one of them, that is, we need to find $\widetilde{G}$ in (3.20). From (3.4) and (3.20), we note that

$$
\mathbf{M}\left(\begin{array}{c}
C \\
-1
\end{array}\right)= \pm 2 \sqrt{D}\left(\begin{array}{c}
-C \\
1
\end{array}\right),
$$

where the upper sign corresponds to the optical case and the lower to the acoustic. Since the quantities $A_{2}, B_{2}$ only involve $F_{Z}$ and $F_{W}$, we write

$$
\widetilde{G}_{1}=i \widetilde{U} F_{Z}+i \widetilde{V} F_{W}
$$


whereupon we find

$$
\begin{aligned}
\widetilde{U} & =\frac{ \pm(-\omega C U+\gamma(1+C) \sin k \cos l)}{\sqrt{D}} \\
\widetilde{V} & =\frac{ \pm(-\omega C V+C \sin l+\gamma(1+C) \sin l \cos k)}{\sqrt{D}} .
\end{aligned}
$$

These quantities are functions of the wavenumbers, and are defined for all $(k, l)$, as illustrated in Figure 7 for the case of $\Omega=1, \gamma=1$. Note that these quantities satisfy the symmetry property $\widetilde{V}(l, k)=\widetilde{U}(k, l)$. From $(3.20)$ with $\widehat{G}=0$, our solutions for $G_{1}, Q_{1}$ are

$$
G_{1}=i C \widetilde{U} F_{Z}+i C \widetilde{V} F_{W}, \quad Q_{1}=-i \widetilde{U} F_{Z}-i \widetilde{V} F_{W}
$$
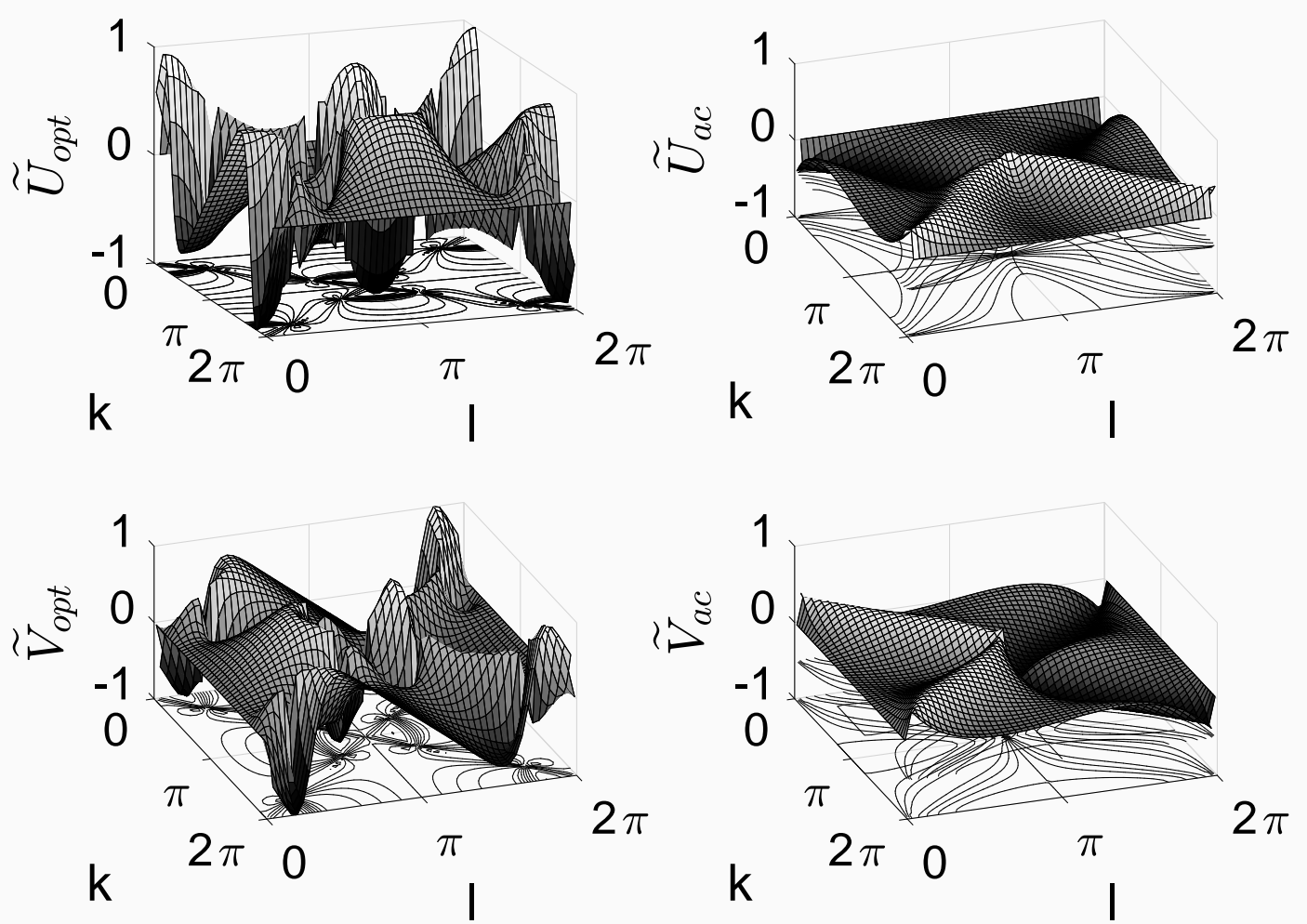

Fig. 7. Plots of the quantities $\widetilde{U}, \widetilde{V}(3.28)-(3.29)$ with $(\Omega, \gamma)=(1,1)$; left panels show the results for the optical mode; and the right panels illustrate the acoustic mode; horizontal velocities $U$ on the top row, vertical velocities, $V$, on the bottom row.

3.6 The second consistency condition and the $N L S-\mathcal{O}\left(\epsilon^{3} e^{i \psi}\right)$ terms

When the ansatz (3.2)-(3.3) is substituted into the governing equations (2.15)(2.16) and terms of $\mathcal{O}\left(\epsilon^{3} \mathrm{e}^{i \psi}\right)$ are equated, we obtain another singular system 
of equations, namely

$$
\mathbf{M}\left(\begin{array}{l}
H_{1} \\
R_{1}
\end{array}\right)=\left(\begin{array}{l}
A_{3} \\
B_{3}
\end{array}\right)
$$

where the matrix $\mathbf{M}$ is again that given in (3.4), and the RHS terms $A_{3}, B_{3}$ are given by equations (A.13)-(A.14) in the appendix.

Since the LHS of (3.31) is again singular, the analysis of this system is similar to (3.17) studied earlier. However, here we only need to derive the consistency condition which $A_{3}, B_{3}$ must satisfy for this system of equations to have a solution. We do not need to find $H_{1}, R_{1}$.

Following the techniques used in Section 3.5, we require $A_{3}+B_{3} C=0$, which produces a single scalar equation for $F$. We use $P=C F$ to eliminate $P$, and (3.16) to rewrite $G_{2}, Q_{2}$ in terms of $F$. We transform to the moving wave coordinates $(Z, W)$ from $(x, y, \tau)$ using (3.21) and terms involving $G_{1}$ and $Q_{1}$ are rewritten using (3.30). The resulting expression for $F$ has the form of a nonlinear Schrödinger equation in $2+1$ dimensions

$$
0=i \Theta F_{T}+D_{Z} F_{Z Z}+D_{W} F_{W W}+D_{M} F_{W Z}+B|F|^{2} F,
$$

where

$$
\begin{aligned}
\Theta= & -2 \omega\left(1+C^{2}\right), \\
D_{Z}= & -U^{2}\left(1+C^{2}\right)+\cos k-2 C \widetilde{U} \sin k+\gamma(1+C)^{2} \cos k \cos l \\
& -2 \gamma\left(C^{2}-1\right) \widetilde{U} \sin k \cos l \\
D_{W}= & -V^{2}\left(1+C^{2}\right)+C^{2} \cos l+2 C \widetilde{V} \sin l+\gamma(1+C)^{2} \cos k \cos l \\
& -2 \gamma\left(C^{2}-1\right) \widetilde{V} \sin l \cos k \\
D_{M}= & -2 U V\left(1+C^{2}\right)-2 C \widetilde{V} \sin k+2 C \widetilde{U} \sin l-2 \gamma(1+C)^{2} \sin k \sin l \\
& -2 \gamma\left(C^{2}-1\right)(\widetilde{U} \sin l \cos k+\widetilde{V} \sin k \cos l),
\end{aligned}
$$

and $B$ given by (A.17). This is the same form of equation as obtained for the scalar two-dimensional lattices analysed previously [8, 9, 33]. To obtain solutions which are localised in both spatial dimensions, we require that the spatial derivatives are elliptic in nature. The alternative is that they are hyperbolic, which gives rise to 'X'-type solutions. Introducing a new variable $\widetilde{W}=D_{M} Z-2 D_{Z} W$, and retaining $Z$, leads to a PDE with only $F_{Z Z}$ and $F_{\widetilde{W} \widetilde{W}}$ derivatives, and no terms in $F_{Z \widetilde{W}}$. For the two second derivatives to have the same sign, we require $4 D_{Z} D_{W}>D_{M}^{2}$, which provides our ellipticity constraint

$$
\mathcal{E}(k, l):=4 D_{Z}(k, l) D_{W}(k, l)-D_{M}(k, l)^{2}>0
$$

In figures 8 and 9 , we plot $\mathcal{E}(k, l)$ for the acoustic and optical cases respectively, showing in black the areas where the constraint is satisfied, that is $\mathcal{E}>0$, and 

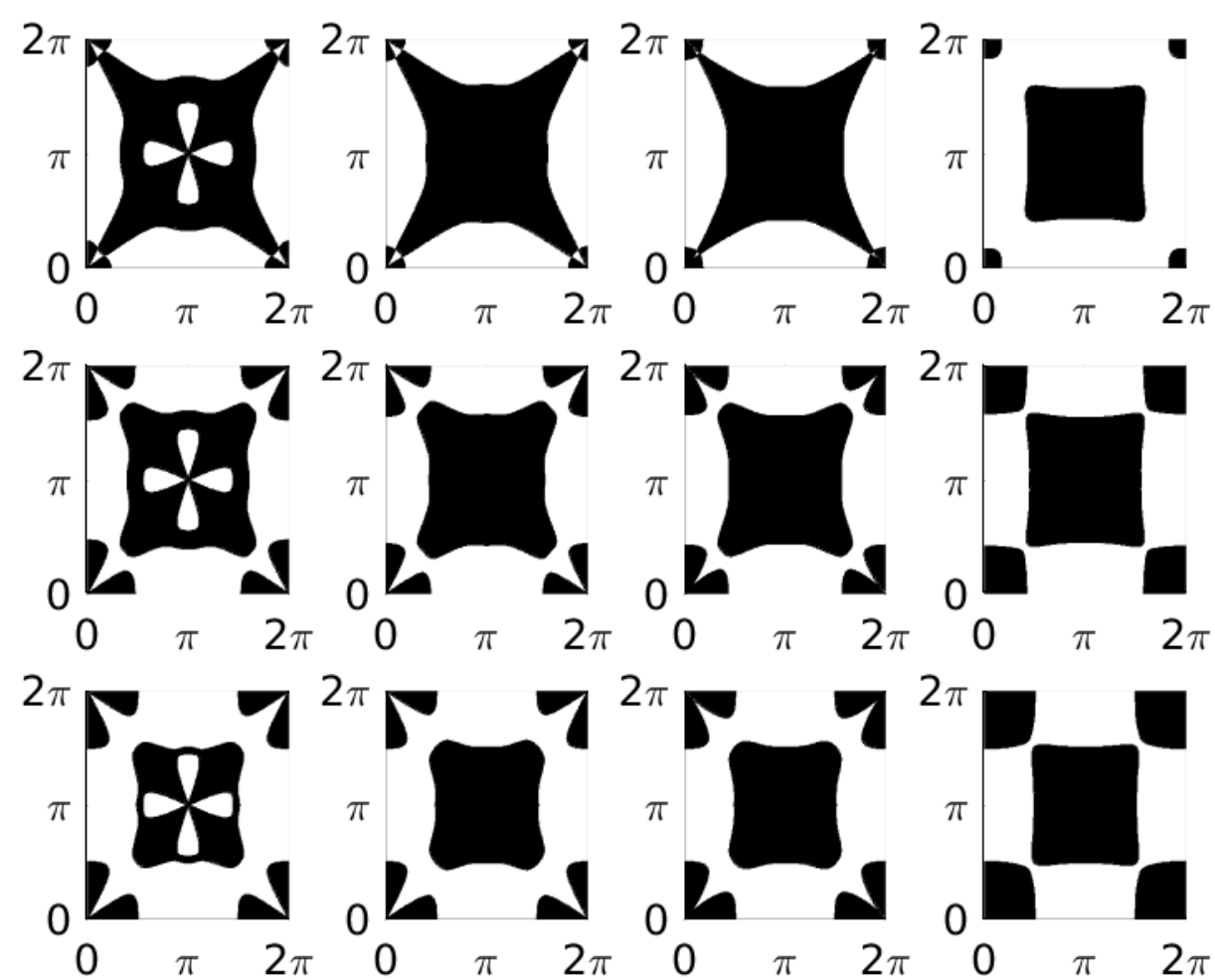

Fig. 8. Plots of the ellipticities $\mathcal{E}(k, l)(3.37)$ for the acoustic mode; in the top row $\Omega=0.1$, in the centre row, $\Omega=1$, and the lowest row, $\Omega=10$. Across each row, $\gamma$ increases, taking the values $\gamma=1 / 8,1 / 2,1,5$. The black areas indicate the regions of $(k, l)$-space where the NLS equation (3.32) is elliptic.

in white, the areas where $\mathcal{E}<0$ and so (3.32) is hyperbolic. The focusing condition for the existence of bright breather modes, namely $D B>0$ is too complicated to be helpful, due to its dependence on so many parameters $(\gamma$, $a, b, h, \lambda, \mu)$ and intermediate expressions $\left(\gamma_{G}, \gamma_{Q}, C, U, V, \widetilde{U}, \widetilde{V}\right)$.

\subsection{Example}

To conclude these calculations, we consider one example case where the wavenumbers are given by $(k, l)=(\pi, 0)$. In appendix A.3 we consider other special wavenumbers, (namely, $(k, l)=(0,0)$ and $(k, l)=(\pi, \pi)$ ), where the calculations of the breather modes lead to inconsistencies. Both here and in Appendix A.3, we calculate and quote simplified explicit expressions for:

- the frequency, $\omega$ given by (3.7),

- the relative amplitudes of the vertical to horizontal displacements, $C=P / F$ given by (3.8)-(3.9), 

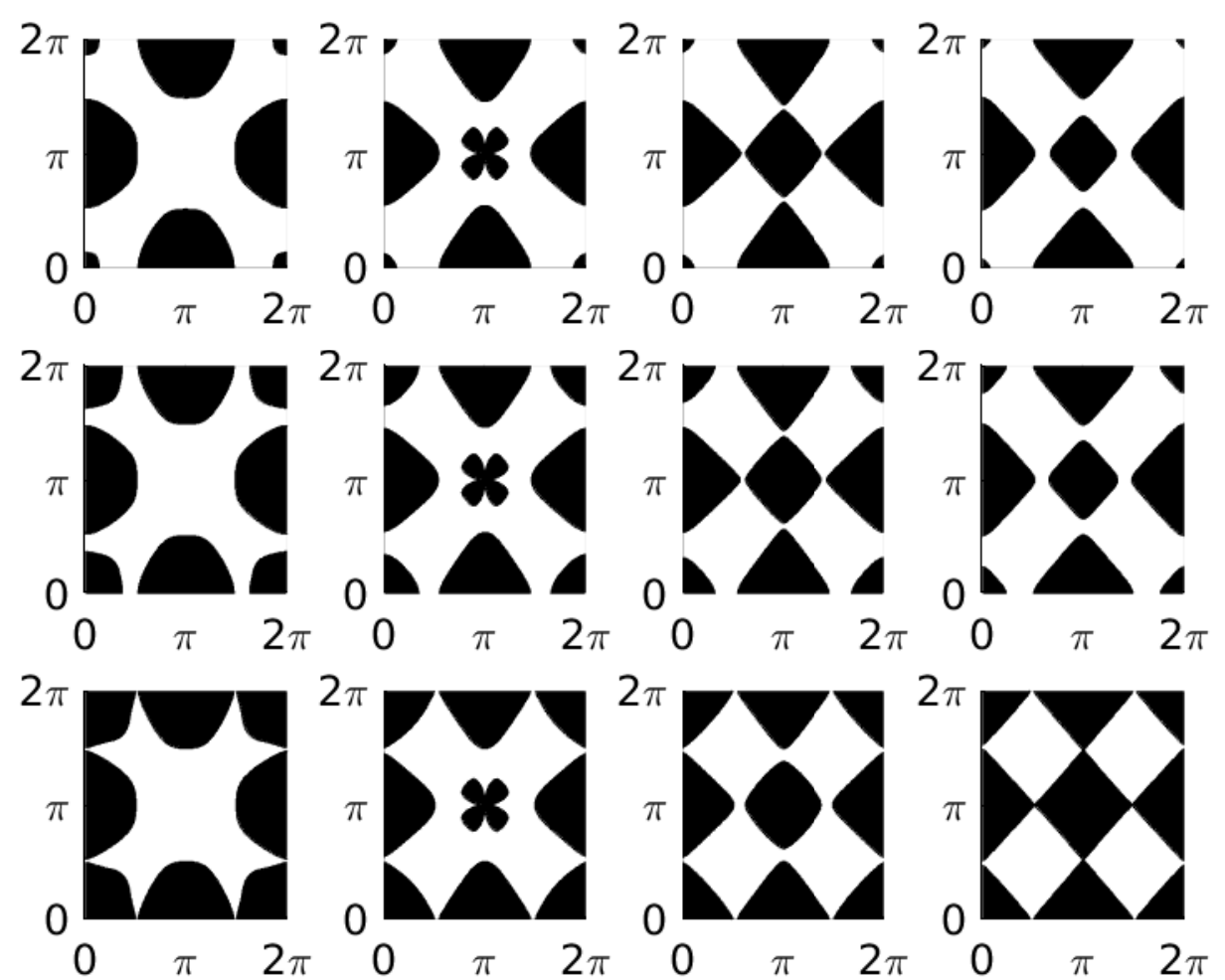

Fig. 9. Plots of the ellipticities $\mathcal{E}(k, l)(3.37)$ for the optical mode; in the top row $\Omega=0.1$, in the centre row, $\Omega=1$, and the lowest row, $\Omega=10$. Across each row, $\gamma$ increases, taking the values $\gamma=1 / 8,1 / 2,1,5$. The black areas indicate the regions of $(k, l)$-space where the NLS equation (3.32) is elliptic.

- the strengths of the second harmonics $\gamma_{G}, \gamma_{Q}$ as defined by (3.16),

- the velocities $U, V$, given by (3.24)-(3.25),

- the correction coefficients $\widetilde{U}, \widetilde{V}$ defined by (3.28)-(3.29),

- the dispersion coefficients, $D_{Z}, D_{W}, D_{M}$ given by (3.34), (3.35), (3.36),

- the ellipticity, $\mathcal{E}$ as defined by (3.37).

At the points $(k, l)=(\pi, 0)$ and $(0, \pi)$ the optical surface of the dispersion equation has its global maximum and the acoustic branch has a saddle point. We write

$$
k=\pi+K \cos \psi, \quad \text { and } \quad l=K \sin \psi
$$

with $0<K \ll 1$; similar results to those quoted below can be obtained for the point $(k, l)=(0, \pi)$ by writing $k=K \cos \psi$ and $l=\pi+K \sin \psi$.

Here the two surfaces are separate and we have 


$$
\omega_{\left\{\begin{array}{l}
\text { opt } \\
\text { ac }
\end{array}\right\}}(\pi, 0)^{2}=\left(\Omega^{2}+2+4 \gamma \pm 2 \sqrt{4 \gamma^{2}+1}\right)-\frac{1}{2} K^{2}\left(\cos 2 \psi+2 \gamma \pm \sqrt{4 \gamma^{2}+1}\right)
$$

together with

$$
C_{\mathrm{opt}}(\pi, 0)=\frac{1+2 \gamma-\sqrt{1+4 \gamma^{2}}}{1-2 \gamma+\sqrt{1+4 \gamma^{2}}}, \quad C_{\mathrm{ac}}(\pi, 0)=\frac{1+2 \gamma+\sqrt{1+4 \gamma^{2}}}{1-2 \gamma-\sqrt{1+4 \gamma^{2}}}
$$

These leading order expressions are well-behaved in the limit $K \rightarrow 0$. As $\gamma$ increases from zero, $C_{\mathrm{opt}}$ rises from zero and plateaus at one. The value of $C_{\mathrm{ac}}$ is always negative (for $\gamma>0$ ) and saturates to the value $C_{\mathrm{ac}} \sim-1$ when $\gamma \gg 1$, and with $C_{\mathrm{ac}}$ diverging to $-\infty$ as $\gamma \rightarrow 0^{+}$. These expressions are illustrated in Figure 10.
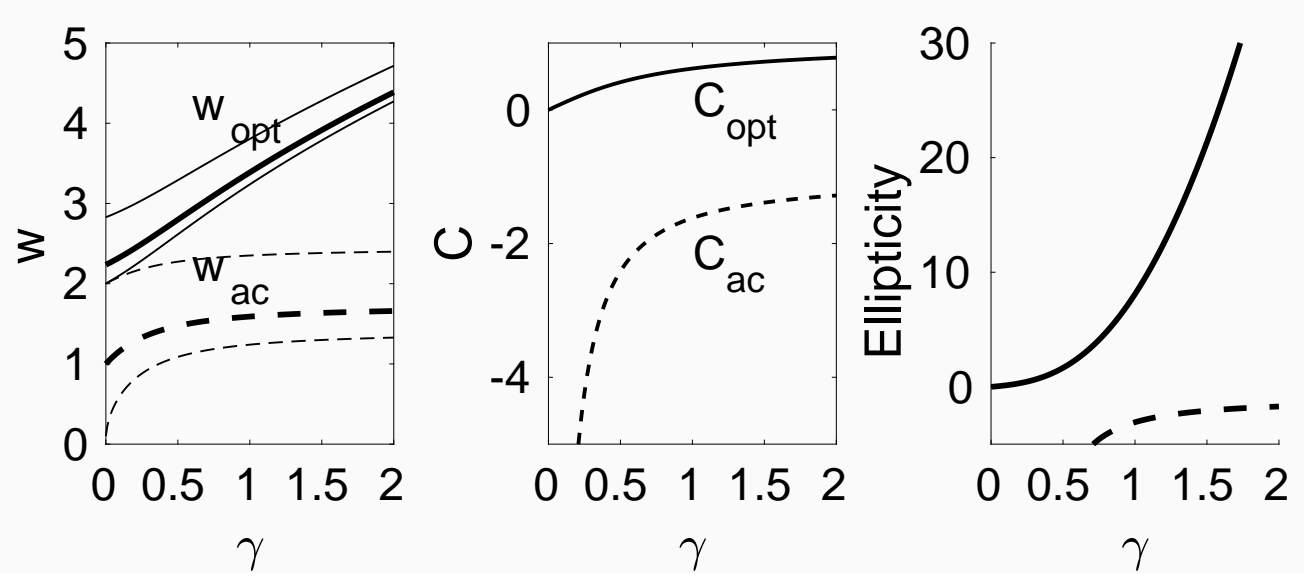

Fig. 10. Left: plots of frequency, $\omega$, against diagonal neighbour coupling strength, $\gamma$ in the case where the wavenumbers are given by $(k, l)=(\pi, 0)$ as given by (3.39). The thick solid curve corresponds to $\omega_{\text {opt }}$ for $\Omega=1$ the thick dashed line to $\omega_{a c}$ for $\Omega=1$, the lower thin solid curve to $\omega_{\text {opt }}$ for $\Omega=0.1$ the lower dashed line to $\omega_{a c}$ for $\Omega=0.1$ the upper thin solid curve to $\omega_{\text {opt }}$ for $\Omega=10$ the upper dashed line to $\omega_{a c}$ for $\Omega=10$. Centre: plots of $C_{\mathrm{ac}}$ and $C_{\mathrm{opt}}$ against $\gamma$ as given by (3.40). We note that $0<C_{\text {opt }}<1$, whilst $C_{a c}<-1$. Right: plots of the ellipticity, $\mathcal{E}$, given by (3.44), where the solid curve represents the optical case and the dashed line the acoustic case.

In this case, we again have $U=V=0$ from (3.24)-(3.25), but this time, the expressions for $\widetilde{U}$ and $\widetilde{V}$ given by (3.28)-(3.29) are well-defined. Since $D=4\left(1+4 \gamma^{2}\right)$, we have $\widetilde{U}=\widetilde{V}=0$, and from (3.20) we have $G_{1}=Q_{1}=0$. Considering the expressions (3.15)-(3.16) for the second harmonic generation, we find $Z_{12}=0$, and $Z_{11}=Z_{22}=\Omega^{2}-4 \omega^{2}, r_{1}=r_{2}=0$ so $G_{2}=Q_{2}=0$. Further simplifications are gained by noting that when $(k, l)=(\pi, 0)$, we have $x \equiv Z$ and $y \equiv W$, together with $\partial_{\tau}=0$. These results significantly simplify the expressions for $A_{3}$ and $B_{3}$, reducing (A.13)-(A.14), to 


$$
\begin{aligned}
A_{3}= & 2 i \omega F_{T}-3|F|^{2} F\left(\lambda+\lambda C^{2}+\mu C^{2}\right)-F_{x x}-48 b|F|^{2} F \\
& -\gamma(1+C)\left(F_{x x}+F_{y y}\right)-\frac{48(a h-1)}{h^{2}}|F|^{2} F C^{2}+\frac{3 \gamma}{h^{2}}|F|^{2} F\left(6+3 C-7 C^{2}\right), \\
B_{3}= & 2 i C \omega F_{T}-3 C|F|^{2} F\left(\lambda C^{2}+\lambda+\mu\right)+C F_{y y}-\frac{24}{h^{2}} C^{3}|F|^{2} F \\
& -\gamma(1+C)\left(F_{x x}+F_{y y}\right)-\frac{48(a h-1)}{h^{2}} C|F|^{2} F+\frac{3 \gamma}{h^{2}}|F|^{2} F\left(3 C^{2}-7 C+2\right) .
\end{aligned}
$$

The solvability condition $A_{3}+C B_{3}=0$ yields the NLS equation (3.32) with $\Theta=2 \omega\left(1+C^{2}\right)$ and

$$
D_{Z}=-1-\gamma(1+C)^{2}, \quad D_{W}=C^{2}-\gamma(1+C)^{2}, \quad D_{M}=0
$$

being the simplified forms of (3.34)-(3.36). The ellipticity, $\mathcal{E}(3.37)$, is thus given by

$$
\mathcal{E}(\pi, 0)=\left[\gamma(1+C)^{2}+1\right]\left[\gamma(1+C)^{2}-C^{2}\right] .
$$

This expression is plotted in the right panel of figure 10. We note that the acoustic case is not elliptic for any choice of $\gamma$, whilst the optical case is elliptic for all choices of $\gamma$. In order to have focusing solutions, we additionally require $D_{Z} B>0$ where $B$ is the coefficient of $|F|^{2} F$ in $A_{3}+B_{3} C=0$ (3.41)-(3.42), which reduces to the NLS being of focusing type provided

$$
0<48 b+\frac{24 C^{2}}{h^{2}}+\frac{96 C^{2}(a h-1)}{h^{2}}+3 \lambda\left(1+C^{2}\right)^{2}+6 \mu C^{2}-\frac{6 \gamma}{h^{2}}\left(3 C^{4}-14 C^{2}+3\right) .
$$

Thus the combined effect of the onsite potential (parameterised by $\lambda, \mu$ ), the diagonal interactions $(\gamma)$ and geometric nonlinearities result in a complicated criterion for the NLS equation to be of the focusing type, even in the case $(k, l)=(\pi, 0)$. In one-dimensional lattices, the inequality usually has the form $b>\nu a^{2}$ for some constant $\nu$; which shows that the coefficient of the cubic nonlinearity has to be large enough to outweigh the square of the quadratic nonlinearity; however, in (3.45), there is no $a^{2}$ term, instead we have several terms which arise due to the geometric nonlinearities of the lattice. With more general wavenumbers $(k, l)$, terms in $a^{2}$ would be generated through the second harmonics $\gamma_{G}, \gamma_{Q} \neq 0$, in additopm to the geometric nonlinearities. Due to the presence of $C$ in (3.45), this expression is really two inequalities - one for each of the acoustic and optical cases and, in general, each is dependent on the wavenumbers $(k, l)$. 


\section{Conclusions}

To summarise, we have used Hamiltonian mechanics to formulate the problem of motion of a two-dimensional lattice under the influence of nonlinear nearest neighbour interactions and an on-site potential. Using a small amplitude expansion, we have obtained the governing equations and found asymptotic approximations for breather modes. Whilst the initial system of equations and the final NLS system are both Hamiltonian, there is nothing special in the asymptotic method of multiple scales which guarantees that the end result will also have a Hamiltonian structure.

The resulting dispersion relation, which describes the properties of linear delocalised waves, has two branches, or sheets, with overlapping frequencies, which share a common minimum, but have differing maxima. There is thus no 'gap' for stable breathers to exist in, but there is a cut off frequency, above which no linear waves can exist. By writing a breather as an envelope solution specifying the amplitude of these linear waves, we have constructed an approximation to a localised moving wave.

At higher orders of the asymptotic calculation, we have found the relative amplitude of motion in the two directions of the lattice, the strengths of second harmonics, the speed of the envelope, and finally the shape of the wave. All these properties of depend on the wavenumbers of the linear wave. There is an ellipticity constraint, which is required for the NLS to be of the elliptic form $i F_{T}=F_{x x}+F_{y y}+B|F|^{2} F$, rather than the hyperbolic form $i F_{T}=F_{x x}-F_{y y}+B|F|^{2}$. This ellipticity constraint is in addition to the inequality $B>0$ on the nonlinearity parameter which is needed for the NLS equation to be of the focusing type rather than the defocusing type. Whilst the dispersion relation for in-plane waves in two-dimensional lattices has been derived by authors in many previously-studied examples, the higher order terms, determining the nonlinear equation for the envelope is the main result of this study. Of particular importance in the NLS equation is the ellipticity constraint which imposes an additional restriction on the wave numbers where long-lived breathers can be expected to exist.

Whilst one of our original aims in this work was to explain the numerical observations of Marin et al. [23, 24] that discrete breathers only travel along the lattice directions, we cannot claim that this work elucidates this behaviour. However, by combining the domains of ellipticity shown in Figures 8 and 9 with the velocities shown in Figure 6, we observe that large portions of the $(k, l)$ space of potential solutions do not lead to breather solutions, in the remaining regions, only a limited range of directions are represented. In future work, we plan to extend these calculations to the triangular and the honeycomb lattices as well as to the more complicated two dimensional FPU lattices. 
ASMA is grateful to Taif University, Saudi Arabia for funding his studies.

\section{A Appendix}

\section{A.1 Details of energy and force expansions for Section 2}

Here we give the full expressions for the small amplitude expansions of the energies and forces which are needed for the derivation of the equations of motion in Section 2. The energies corresponding to the horizontal, vertical and diagonal springs, which appear in the Hamiltonian (2.13) are given by

$$
\begin{aligned}
V_{h}= & \frac{1}{2}\left(u_{m+1, n}-u_{m, n}\right)^{2}+\frac{1}{2 h}\left(u_{m+1, n}-u_{m, n}\right)\left(v_{m+1, n}-v_{m, n}\right)^{2} \\
& +\frac{1}{8 h^{2}}\left(v_{m+1, n}-v_{m, n}\right)^{4}+\left(\frac{a h-1}{2 h^{2}}\right)\left(u_{m+1, n}-u_{m, n}\right)^{2}\left(v_{m+1, n}-v_{m, n}\right)^{2} \\
& +\frac{1}{3} a\left(u_{m+1, n}-u_{m, n}\right)^{3}+\frac{1}{4} b\left(u_{m+1, n}-u_{m, n}\right)^{4} \\
V_{v}= & \frac{1}{2}\left(v_{m, n+1}-v_{m, n}\right)^{2}+\frac{1}{2 h}\left(u_{m, n+1}-u_{m, n}\right)^{2}\left(v_{m, n+1}-v_{m, n}\right) \\
& +\frac{1}{8 h^{2}}\left(u_{m, n+1}-u_{m, n}\right)^{4}+\left(\frac{a h-1}{2 h^{2}}\right)\left(u_{m, n+1}-u_{m, n}\right)^{2}\left(v_{m, n+1}-v_{m, n}\right)^{2} \\
& +\frac{1}{3} a\left(v_{m, n+1}-v_{m, n}\right)^{3}+\frac{1}{4} b\left(v_{m, n+1}-v_{m, n}\right)^{4}, \\
V_{d}= & \frac{1}{4} \gamma\left(u_{m+1, n+1}-u_{m, n}\right)^{2}+\frac{1}{2} \gamma\left(u_{m+1, n+1}-u_{m, n}\right)\left(v_{m+1, n+1}-v_{m, n}\right) \\
& +\frac{\gamma}{8 h}\left(u_{m+1, n+1}-u_{m, n}\right)^{3}-\frac{\gamma}{8 h}\left(u_{m+1, n+1}-u_{m, n}\right)^{2}\left(v_{m+1, n+1}-v_{m, n}\right) \\
& -\frac{\gamma}{8 h}\left(u_{m+1, n+1}-u_{m, n}\right)\left(v_{m+1, n+1}-v_{m, n}\right)^{2}+\frac{\gamma}{8 h}\left(v_{m+1, n+1}-v_{m, n}\right)^{3} \\
& +\frac{7 \gamma}{32 h^{2}}\left(u_{m+1, n+1}-u_{m, n}\right)^{2}\left(v_{m+1, n+1}-v_{m, n}\right)^{2}+\frac{1}{4} \gamma\left(v_{m+1, n+1}-v_{m, n}\right)^{2} \\
& -\frac{\gamma}{16 h^{2}}\left(u_{m+1, n+1}-u_{m, n}\right)^{3}\left(v_{m+1, n+1}-v_{m, n}\right)-\frac{3 \gamma}{64 h^{2}}\left(u_{m+1, n+1}-u_{m, n}\right)^{4} \\
& -\frac{\gamma}{16 h^{2}}\left(u_{m+1, n+1}-u_{m, n}\right)\left(v_{m+1, n+1}-v_{m, n}\right)^{3}-\frac{3 \gamma}{64 h^{2}}\left(v_{m+1, n+1}-v_{m, n}\right)^{4}(\mathrm{~A} .3) \\
& \\
&
\end{aligned}
$$




$$
\begin{aligned}
V_{e}= & \frac{1}{4} \gamma\left(u_{m+1, n-1}-u_{m, n}\right)^{2}-\frac{1}{2} \gamma\left(u_{m+1, n-1}-u_{m, n}\right)\left(v_{m+1, n-1}-v_{m, n}\right) \\
& +\frac{\gamma}{8 h}\left(u_{m+1, n-1}-u_{m, n}\right)^{3}+\frac{\gamma}{8 h}\left(u_{m+1, n-1}-u_{m, n}\right)^{2}\left(v_{m+1, n-1}-v_{m, n}\right) \\
& -\frac{\gamma}{8 h}\left(u_{m+1, n-1}-u_{m, n}\right)\left(v_{m+1, n-1}-v_{m, n}\right)^{2}-\frac{\gamma}{8 h}\left(v_{m+1, n-1}-v_{m, n}\right)^{3} \\
& +\frac{7 \gamma}{32 h^{2}}\left(u_{m+1, n-1}-u_{m, n}\right)^{2}\left(v_{m+1, n-1}-v_{m, n}\right)^{2}+\frac{1}{4} \gamma\left(v_{m+1, n-1}-v_{m, n}\right)^{2} \\
& +\frac{\gamma}{16 h^{2}}\left(u_{m+1, n-1}-u_{m, n}\right)^{3}\left(v_{m+1, n-1}-v_{m, n}\right)-\frac{3 \gamma}{64 h^{2}}\left(u_{m+1, n-1}-u_{m, n}\right)^{4} \\
& +\frac{\gamma}{16 h^{2}}\left(u_{m+1, n-1}-u_{m, n}\right)\left(v_{m+1, n-1}-v_{m, n}\right)^{3}-\frac{3 \gamma}{64 h^{2}}\left(v_{m+1, n-1}-v_{m, n}\right)^{4}(. \mathrm{A} .4)
\end{aligned}
$$

The corresponding forces in the equations of motion for $u_{m, n}$ and $v_{m, n}(2.15)-$ (2.16) are given by

$$
\begin{aligned}
F_{h}= & \left(u_{m+1, n}-2 u_{m, n}+u_{m-1, n}\right)+\frac{1}{2 h}\left[\left(v_{m+1, n}-v_{m, n}\right)^{2}-\left(v_{m, n}-v_{m-1, n}\right)^{2}\right] \\
& +a\left[\left(u_{m+1, n}-u_{m, n}\right)^{2}-\left(u_{m, n}-u_{m-1, n}\right)^{2}\right] \\
& +b\left[\left(u_{m+1, n}-u_{m, n}\right)^{3}-\left(u_{m, n}-u_{m-1, n}\right)^{3}\right] \\
& +\left(\frac{a h-1}{h^{2}}\right)\left[\left(u_{m+1, n}-u_{m, n}\right)\left(v_{m+1, n}-v_{m, n}\right)^{2}-\left(u_{m, n}-u_{m-1, n}\right)\left(v_{m, n}-v_{m-1, n}\right)^{2}\right],
\end{aligned}
$$

$$
\begin{aligned}
F_{v}= & \frac{1}{h}\left[\left(v_{m, n+1}-v_{m, n}\right)\left(u_{m, n+1}-u_{m, n}\right)-\left(v_{m, n}-v_{m, n-1}\right)\left(u_{m, n}-u_{m, n-1}\right)\right] \\
& +\frac{1}{2 h^{2}}\left[\left(u_{m, n+1}-u_{m, n}\right)^{3}-\left(u_{m, n}-u_{m, n-1}\right)^{3}\right] \\
& +\left(\frac{a h-1}{h^{2}}\right)\left[\left(u_{m, n+1}-u_{m, n}\right)\left(v_{m, n+1}-v_{m, n}\right)^{2}-\left(u_{m, n}-u_{m, n-1}\right)\left(v_{m, n}-v_{m, n-1}\right)^{2}\right],
\end{aligned}
$$




$$
\begin{aligned}
F_{d}= & \frac{1}{2} \gamma\left[\left(u_{m+1, n+1}-2 u_{m, n}+u_{m-1, n-1}\right)+\left(v_{m+1, n+1}-2 v_{m, n}+v_{m-1, n-1}\right)\right] \\
& +\frac{3 \gamma}{8 h}\left[\left(u_{m+1, n+1}-u_{m, n}\right)^{2}-\left(u_{m, n}-u_{m-1, n-1}\right)^{2}\right] \\
& -\frac{3 \gamma}{16 h^{2}}\left[\left(u_{m+1, n+1}-u_{m, n}\right)^{3}-\left(u_{m, n}-u_{m-1, n-1}\right)^{3}\right] \\
& -\frac{\gamma}{4 h}\left[\left(u_{m+1, n+1}-u_{m, n}\right)\left(v_{m+1, n+1}-v_{m, n}\right)-\left(u_{m, n}-u_{m-1, n-1}\right)\left(v_{m, n}-v_{m-1, n-1}\right)\right] \\
& -\frac{\gamma}{8 h}\left[\left(v_{m+1, n+1}-v_{m, n}\right)^{2}-\left(v_{m, n}-v_{m-1, n-1}\right)^{2}\right] \\
& -\frac{3 \gamma}{16 h^{2}}\left[\left(u_{m+1, n+1}-u_{m, n}\right)^{2}\left(v_{m+1, n+1}-v_{m, n}\right)-\left(u_{m, n}-u_{m-1, n-1}\right)^{2}\left(v_{m, n}-v_{m-1, n-1}\right)\right] \\
& +\frac{7 \gamma}{16 h^{2}}\left[\left(u_{m+1, n+1}-u_{m, n}\right)\left(v_{m+1, n+1}-v_{m, n}\right)^{2}-\left(u_{m, n}-u_{m-1, n-1}\right)\left(v_{m, n}-v_{m-1, n-1}\right)^{2}\right] \\
& -\frac{\gamma}{16 h^{2}}\left[\left(v_{m+1, n+1}-v_{m, n}\right)^{3}-\left(v_{m, n}-v_{m-1, n-1}\right)^{3}\right],
\end{aligned}
$$

$$
\begin{aligned}
F_{e}= & \frac{1}{2} \gamma\left[\left(u_{m+1, n-1}-2 u_{m, n}+u_{m-1, n+1}\right)+\left(v_{m+1, n-1}-2 v_{m, n}+v_{m-1, n+1}\right)\right] \\
& +\frac{3 \gamma}{8 h}\left[\left(u_{m+1, n-1}-u_{m, n}\right)^{2}-\left(u_{m, n}-u_{m-1, n+1}\right)^{2}\right] \\
& -\frac{3 \gamma}{16 h^{2}}\left[\left(u_{m+1, n-1}-u_{m, n}\right)^{3}-\left(u_{m, n}-u_{m-1, n+1}\right)^{3}\right] \\
& +\frac{\gamma}{4 h}\left[\left(u_{m+1, n-1}-u_{m, n}\right)\left(v_{m+1, n-1}-v_{m, n}\right)-\left(u_{m, n}-u_{m-1, n+1}\right)\left(v_{m, n}-v_{m-1, n+1}\right)\right] \\
& +\frac{\gamma}{8 h}\left[\left(v_{m+1, n-1}-v_{m, n}\right)^{2}-\left(v_{m, n}-v_{m-1, n+1}\right)^{2}\right] \\
& +\frac{3 \gamma}{16 h^{2}}\left[\left(u_{m+1, n-1}-u_{m, n}\right)^{2}\left(v_{m+1, n-1}-v_{m, n}\right)-\left(u_{m, n}-u_{m-1, n+1}\right)^{2}\left(v_{m, n}-v_{m-1, n+1}\right)\right] \\
& +\frac{7 \gamma}{16 h^{2}}\left[\left(u_{m+1, n-1}-u_{m, n}\right)\left(v_{m+1, n-1}-v_{m, n}\right)^{2}-\left(u_{m, n}-u_{m-1, n+1}\right)\left(v_{m, n}-v_{m-1, n+1}\right)^{2}\right] \\
& +\frac{\gamma}{16 h^{2}}\left[\left(v_{m+1, n-1}-v_{m, n}\right)^{3}-\left(v_{m, n}-v_{m-1, n+1}\right)^{3}\right]
\end{aligned}
$$

$$
\begin{aligned}
G_{h}= & \frac{1}{h}\left[\left(u_{m+1, n}-u_{m, n}\right)\left(v_{m+1, n}-v_{m, n}\right)-\left(u_{m, n}-u_{m-1, n}\right)\left(v_{m, n}-v_{m-1, n}\right)\right] \\
& +\left(\frac{a h-1}{h^{2}}\right)\left[\left(u_{m+1, n}-u_{m, n}\right)^{2}\left(v_{m+1, n}-v_{m, n}\right)-\left(u_{m, n}-u_{m-1, n}\right)^{2}\left(v_{m, n}-v_{m-1, n}\right)\right] \\
& +\frac{1}{2 h^{2}}\left[\left(v_{m+1, n}-v_{m, n}\right)^{3}-\left(v_{m, n}-v_{m-1, n}\right)^{3}\right]
\end{aligned}
$$




$$
\begin{aligned}
G_{v}= & \left(v_{m, n+1}-2 v_{m, n}+v_{m, n-1}\right)+\frac{1}{2 h}\left[\left(u_{m, n+1}-u_{m, n}\right)^{2}-\left(u_{m, n}-u_{m, n-1}\right)^{2}\right] \\
& +a\left[\left(v_{m, n+1}-v_{m, n}\right)^{2}-\left(v_{m, n}-v_{m, n-1}\right)^{2}\right]+b\left[\left(v_{m, n+1}-v_{m, n}\right)^{3}-\left(v_{m, n}-v_{m, n-1}\right)^{3}\right] \\
& +\left(\frac{a h-1}{h^{2}}\right)\left[\left(u_{m, n+1}-u_{m, n}\right)^{2}\left(v_{m, n+1}-v_{m, n}\right)-\left(u_{m, n}-u_{m, n-1}\right)^{2}\left(v_{m, n}-v_{m, n-1}\right)\right],
\end{aligned}
$$

$$
\begin{aligned}
G_{d}= & \frac{1}{2} \gamma\left[\left(v_{m+1, n+1}-2 v_{m, n}+v_{m-1, n-1}\right)+\left(u_{m+1, n+1}-2 u_{m, n}+u_{m-1, n-1}\right)\right] \\
& +\frac{3 \gamma}{8 h}\left[\left(v_{m+1, n+1}-v_{m, n}\right)^{2}-\left(v_{m, n}-v_{m-1, n-1}\right)^{2}\right] \\
& -\frac{\gamma}{8 h}\left[\left(u_{m+1, n+1}-u_{m, n}\right)^{2}-\left(u_{m, n}-u_{m-1, n-1}\right)^{2}\right] \\
& -\frac{\gamma}{4 h}\left[\left(u_{m+1, n+1}-u_{m, n}\right)\left(v_{m+1, n+1}-v_{m, n}\right)-\left(u_{m, n}-u_{m-1, n-1}\right)\left(v_{m, n}-v_{m-1, n-1}\right)\right] \\
& +\frac{7 \gamma}{16 h^{2}}\left[\left(u_{m+1, n+1}-u_{m, n}\right)^{2}\left(v_{m+1, n+1}-v_{m, n}\right)-\left(u_{m, n}-u_{m-1, n-1}\right)^{2}\left(v_{m, n}-v_{m-1, n-1}\right)\right] \\
& -\frac{3 \gamma}{16 h^{2}}\left[\left(u_{m+1, n+1}-u_{m, n}\right)\left(v_{m+1, n+1}-v_{m, n}\right)^{2}-\left(u_{m, n}-u_{m-1, n-1}\right)\left(v_{m, n}-v_{m-1, n-1}\right)^{2}\right] \\
& -\frac{3 \gamma}{16 h^{2}}\left[\left(v_{m+1, n+1}-v_{m, n}\right)^{3}-\left(v_{m, n}-v_{m-1, n-1}\right)^{3}\right] \\
& -\frac{\gamma}{16 h^{2}}\left[\left(u_{m+1, n+1}-u_{m, n}\right)^{3}-\left(u_{m, n}-u_{m-1, n-1}\right)^{3}\right]
\end{aligned}
$$$$
G_{e}=\frac{1}{2} \gamma\left[\left(v_{m+1, n-1}-2 v_{m, n}+v_{m-1, n+1}\right)+\left(u_{m+1, n-1}-2 u_{m, n}+u_{m-1, n+1}\right)\right]
$$$$
-\frac{\gamma}{4 h}\left[\left(u_{m+1, n-1}-u_{m, n}\right)\left(v_{m+1, n-1}-v_{m, n}\right)-\left(u_{m, n}-u_{m-1, n+1}\right)\left(v_{m, n}-v_{m-1, n+1}\right)\right]
$$$$
+\frac{\gamma}{8 h}\left[\left(u_{m+1, n-1}-u_{m, n}\right)^{2}-\left(u_{m, n}-u_{m-1, n+1}\right)^{2}\right]
$$$$
-\frac{3 \gamma}{8 h}\left[\left(v_{m+1, n-1}-v_{m, n}\right)^{2}-\left(v_{m, n}-v_{m-1, n+1}\right)^{2}\right]
$$$$
+\frac{7 \gamma}{16 h^{2}}\left[\left(u_{m+1, n-1}-u_{m, n}\right)^{2}\left(v_{m+1, n-1}-v_{m, n}\right)-\left(u_{m, n}-u_{m-1, n+1}\right)^{2}\left(v_{m, n}-v_{m-1, n+1}\right)\right]
$$$$
+\frac{3 \gamma}{16 h^{2}}\left[\left(u_{m+1, n-1}-u_{m, n}\right)\left(v_{m+1, n-1}-v_{m, n}\right)^{2}-\left(u_{m, n}-u_{m-1, n+1}\right)\left(v_{m, n}-v_{m-1, n+1}\right)^{2}\right]
$$$$
+\frac{\gamma}{16 h^{2}}\left[\left(u_{m+1, n-1}-u_{m, n}\right)^{3}-\left(u_{m, n}-u_{m-1, n+1}\right)^{3}\right]
$$$$
-\frac{3 \gamma}{16 h^{2}}\left[\left(v_{m+1, n-1}-v_{m, n}\right)^{3}-\left(v_{m, n}-v_{m-1, n+1}\right)^{3}\right] \text {. }
$$

\section{A.2 RHS of (3.6) in $\mathcal{O}\left(\epsilon^{3} e^{i \psi}\right)$ equations}

The equations for the RHS of the governing equations at $\mathcal{O}\left(\epsilon^{3} \mathrm{e}^{i \psi}\right)$ from section 3.6, where we seek the consistency condition for $\mathbf{M}\left(H_{1}, R_{1}\right)^{T}=\left(A_{3}, B_{3}\right)^{T}$ to 
have solutions, are given by

$$
\begin{aligned}
& A_{3}=-2 i \omega F_{T}-F_{\tau \tau}-2 i \omega G_{1, \tau}-3 \lambda|F|^{2} F-(\lambda+\mu)\left(2|P|^{2} F+P^{2} F^{*}\right) \\
& +F_{x x} \cos k+2 i \sin k G_{1, x}-48 b|F|^{2} F \sin ^{4} \frac{1}{2} k+32 i a G_{2} F^{*} \sin ^{3} \frac{1}{2} k \cos \frac{1}{2} k \\
& -\frac{16(a h-1)}{h^{2}}\left(P^{2} F^{*}+2|P|^{2} F\right)\left(\sin ^{4} \frac{1}{2} k+\sin ^{4} \frac{1}{2} l\right)+\frac{16 i}{h} Q_{2} P^{*} \sin ^{3} \frac{1}{2} k \cos \frac{1}{2} k \\
& -\frac{24}{h^{2}}|F|^{2} F \sin ^{4} \frac{1}{2} l+\frac{16 i}{h}\left(G_{2} P^{*}+Q_{2} F^{*}\right) \sin ^{3} \frac{1}{2} l \cos \frac{1}{2} l \\
& +\gamma\left(F_{x x}+F_{y y}+P_{x x}+P_{y y}\right) \cos k \cos l-2 \gamma\left(F_{x y}+P_{x y}\right) \sin k \sin l \\
& +2 \gamma i\left(G_{1, x}+Q_{1, x}\right) \sin k \cos l+2 \gamma i\left(G_{1, y}+Q_{1, y}\right) \sin l \cos k \\
& +\frac{\gamma}{h^{2}}\left(9|F|^{2} F+3|P|^{2} P+3 F^{2} P^{*}+6|F|^{2} P-7 P^{2} F^{*}-14|P|^{2} F\right) \sin ^{4} \frac{1}{2}(k+l) \\
& +\frac{\gamma}{h^{2}}\left(9|F|^{2} F-3|P|^{2} P-3 F^{2} P^{*}-6|F|^{2} P-7 P^{2} F^{*}-14|P|^{2} F\right) \sin ^{4} \frac{1}{2}(k-l) \\
& +\frac{4 i \gamma}{h}\left(3 G_{2} F^{*}-G_{2} P^{*}-Q_{2} F^{*}-Q_{2} P^{*}\right) \sin ^{3} \frac{1}{2}(k+l) \cos \frac{1}{2}(k+l) \\
& +\frac{4 i \gamma}{h}\left(G_{2} P^{*}+Q_{2} F^{*}+3 G_{2} F^{*}+Q_{2} P^{*}\right) \sin ^{3} \frac{1}{2}(k-l) \cos \frac{1}{2}(k-l), \\
& B_{3}=-2 i \omega P_{T}-P_{\tau \tau}-2 i \omega Q_{1, \tau}-3 \lambda|P|^{2} P-(\lambda+\mu)\left(2|F|^{2} P-F^{2} P^{*}\right) \\
& +P_{y y} \cos l+2 i \sin l Q_{1, y}-48 b|P|^{2} P \sin ^{4} \frac{1}{2} l+32 i a Q_{2} P^{*} \sin ^{3} \frac{1}{2} l \cos \frac{1}{2} l \\
& -\frac{16(a h-1)}{h^{2}}\left(F^{2} P^{*}+2|F|^{2} P\right)\left(\sin ^{4} \frac{1}{2} l+\sin ^{4} \frac{1}{2} k\right)+\frac{16 i}{h} G_{2} F^{*} \sin ^{3} \frac{1}{2} l \cos \frac{1}{2} l \\
& -\frac{24}{h^{2}}|P|^{2} P \sin ^{4} \frac{1}{2} k+\frac{16 i}{h}\left(Q_{2} F^{*}+G_{2} P^{*}\right) \sin ^{3} \frac{1}{2} k \cos \frac{1}{2} k \\
& +\gamma\left(F_{x x}+F_{y y}+P_{x x}+P_{y y}\right) \cos k \cos l-2 \gamma\left(F_{x y}+P_{x y}\right) \sin l \sin k \\
& +2 \gamma i\left(G_{1, x}+Q_{1, x}\right) \sin k \cos l+2 \gamma i\left(G_{1, y}+Q_{1, y}\right) \sin l \cos k \\
& +\frac{\gamma}{h^{2}}\left(9|P|^{2} P+3|F|^{2} F+3 P^{2} F^{*}+6|P|^{2} F-7 F^{2} P^{*}-14|F|^{2} P\right) \sin ^{4} \frac{1}{2}(k+l) \\
& +\frac{\gamma}{h^{2}}\left(9|P|^{2} P-3|F|^{2} F-3 P^{2} F^{*}-6|P|^{2} F-7 F^{2} P^{*}-14|F|^{2} P\right) \sin ^{4} \frac{1}{2}(k-l) \\
& +\frac{4 i \gamma}{h}\left(3 Q_{2} P^{*}-Q_{2} F^{*}-G_{2} P^{*}-G_{2} F^{*}\right) \sin ^{3} \frac{1}{2}(k+l) \cos \frac{1}{2}(k+l) \\
& -\frac{4 i \gamma}{h}\left(Q_{2} F^{*}+G_{2} P^{*}+3 Q_{2} P^{*}+G_{2} F^{*}\right) \sin ^{3} \frac{1}{2}(k-l) \cos \frac{1}{2}(k-l),
\end{aligned}
$$

where $B_{3}$ can be obtained from $A_{3}$ by making the transpositions $k \leftrightarrow l, x \leftrightarrow y$, $F \leftrightarrow P, G_{1} \leftrightarrow Q_{1}, G_{2} \leftrightarrow Q_{2}$.

We simplify these expressions for $A_{3}, B_{3}$ by making use of (3.8)-(3.9), (3.16), (3.30), (3.21), namely, $P=C F, G_{2}=\gamma_{G} F^{2}, Q_{2}=\gamma_{Q} F^{2}, G_{1}=i C \widetilde{U} F_{Z}+$ $i C \widetilde{V} F_{W}, Q_{1}=-i \widetilde{U} F_{Z}-i \widetilde{V} F_{W}, \partial_{x}=\partial_{Z}, \partial_{y}=\partial_{W}, \partial_{\tau}=-U \partial_{Z}-V \partial_{W}$ which imply 


$$
\begin{aligned}
G_{1, \tau} & \left.=-i C U \widetilde{U} F_{Z Z}-i C V \widetilde{V} F_{W W}-i C(U \widetilde{V}+V \widetilde{U}) F_{W Z}\right) \\
Q_{1, \tau} & =i U \widetilde{U} F_{Z Z}+i V \widetilde{V} F_{W W}+i(U \widetilde{V}+V \widetilde{U}) F_{W Z}
\end{aligned}
$$

Taking all the equations in this subsection together with those in section 3.6, yields the PDE (3.32).

Finally, we quote the coefficient of nonlinearity, $B$, in (3.32), is given by

$$
\begin{aligned}
B= & -6 C^{2} \mu^{2}-3 \lambda\left(C^{2}+1\right)^{2}-48 b\left(\sin ^{4} \frac{1}{2} k+C^{4} \sin ^{4} \frac{1}{2} l\right) \\
& +32 a i\left(\gamma_{G} \sin ^{3} \frac{1}{2} k \cos \frac{1}{2} k+\gamma_{Q} C^{2} \sin ^{3} \frac{1}{2} l \cos \frac{1}{2} l\right) \\
& +\frac{16 C i}{h}\left(\gamma_{Q} \sin ^{3} \frac{1}{2} k \cos \frac{1}{2} k+\gamma_{G} \sin ^{3} \frac{1}{2} l \cos \frac{1}{2} l\right) \\
& -\frac{96(a h-1) C^{2}}{h^{2}}\left(\sin ^{4} \frac{1}{2} k+\sin ^{4} \frac{1}{2} l\right)-\frac{24}{h^{2}}\left(\sin ^{4} \frac{1}{2} l+C^{4} \sin ^{4} \frac{1}{2} k\right) \\
& +\frac{16 i}{h}\left(\gamma_{Q}+C \gamma_{G}\right)\left(C \sin ^{3} \frac{1}{2} k \cos \frac{1}{2} k+\sin ^{3} \frac{1}{2} l \cos \frac{1}{2} l\right) \\
& +3 \gamma(3 C+1)(C+3)(C-1)^{2} \sin ^{4} \frac{1}{2}(k+l) \\
& +3 \gamma(3 C-1)(C-3)(C+1)^{2} \sin ^{4} \frac{1}{2}(k-l) \\
& +\frac{4 i \gamma}{h}\left[(3 C+1) \gamma_{Q}-\gamma_{G}(C+3)\right](C-1) \sin ^{3} \frac{1}{2}(k+l) \cos \frac{1}{2}(k+l) \\
& +\frac{4 i \gamma}{h}\left[\left(3-C^{2}\right) \gamma_{G}+\gamma_{Q}\left(1-3 C^{2}\right)\right] \sin ^{3} \frac{1}{2}(k-l) \cos \frac{1}{2}(k-l),
\end{aligned}
$$

which we note is real, since the quantities $r_{1}$ and $r_{2}$ given by (3.13)-(3.14) are pure imaginary, and so the parameters $\gamma_{G}, \gamma_{Q}$ are also both pure imaginary, being defined by (3.16).

\section{A.3 Example expansions for certain wavenumbers}

In this section we consider the expansions quoted in Section 3, for certain special values of the wavenumbers, $(k, l)$. We use the stationary points of the dispersion surfaces as examples, since breathers that bifurcate from these points have the potential to have frequencies different from any linear wave. We aim to follow the calculations listed at the start of Section 3.7.

\section{A.3.1 Global minimum of dispersion relation, $(k, l)=(0,0)$}

To investigate the behaviour of the system as we approach the global minimum of the dispersion relation at $(k, l)=(0,0)$ we put

$$
k=K \cos \psi, \quad \text { and } \quad l=K \sin \psi,
$$


and consider the asymptotic limit $K \rightarrow 0^{+}(0<K \ll 1)$. As noted in Table 1 , both the acoustic and optical surfaces have a minimum at this point.

Determining the frequency from (3.7), we obtain

$$
\omega_{\left\{\begin{array}{l}
\text { opt } \\
\text { ac }
\end{array}\right\}}^{2}(0,0)=\Omega^{2}+K^{2}\left(\gamma+\frac{1}{2} \pm \sqrt{\gamma^{2}+\frac{1}{4} \cos ^{2}(2 \psi)}\right)
$$

so the frequencies are well-defined in this limit as $\omega=\Omega$; however, the leading order expressions for the ratio of vertical to horizontal displacements, $C$, as given by (3.8) and (3.9) are

$$
\begin{aligned}
C_{\text {opt }}(0,0) & =\frac{\cos (2 \psi)+2 \gamma-\sqrt{4 \gamma^{2}+\cos ^{2}(2 \psi)}}{\cos (2 \psi)-2 \gamma+\sqrt{4 \gamma^{2}+\cos ^{2}(2 \psi)}}, \\
C_{\mathrm{ac}}(0,0) & =\frac{-2 \gamma-\cos (2 \psi)-\sqrt{4 \gamma^{2}+\cos ^{2}(2 \psi)}}{2 \gamma-\cos (2 \psi)+\sqrt{4 \gamma^{2}+\cos ^{2}(2 \psi)}} .
\end{aligned}
$$

Since both these expressions depend on $\psi$ at leading order in $K \ll 1$, the value of $C$ is not well-defined for either acoustic or optical modes. Furthermore, whilst (3.24)-(3.25) give $U=V=0$ when $k=l=0$, the expressions for $\widetilde{U}, \widetilde{V}$, (3.28)-(3.29) both diverge as $k, l \rightarrow 0$ (since $D=\mathcal{O}\left(K^{4}\right)$ as given by (3.7)).

\section{A.3.2 Max $/$ min of dispersion relation, $(k, l)=(\pi, \pi)$}

Next, we consider the behaviour of the system in the limit of $K \rightarrow 0^{+}(0<$ $K \ll 1)$ where

$$
k=\pi+K \cos \psi, \quad \text { and } \quad l=\pi+K \sin \psi,
$$

in which limit, the optical surface has a local minimum, and the acoustic surface experiences its global maximum. Here, the surfaces meet and we find the frequency $\omega$ is given by

$$
\omega_{\left\{\begin{array}{l}
\text { opt } \\
\text { ac }
\end{array}\right\}}^{2}(\pi, \pi)=\left(\Omega^{2}+4\right)+K^{2}\left(\gamma-\frac{1}{2} \pm \sqrt{\gamma^{2}+\frac{1}{4} \cos ^{2}(2 \psi)}\right)
$$

which, in the limit $K \rightarrow 0$, is well-defined as $\omega=\sqrt{\Omega^{2}+4}$. However, the leading order expressions for $C$ are 


$$
\begin{aligned}
C_{\mathrm{opt}}(\pi, \pi) & =\frac{2 \gamma-\cos (2 \psi)-\sqrt{4 \gamma^{2}+\cos ^{2}(2 \psi)}}{-2 \gamma-\cos (2 \psi)+\sqrt{4 \gamma^{2}+\cos ^{2}(2 \psi)}}, \\
C_{\mathrm{ac}}(\pi, \pi) & =\frac{-2 \gamma+\cos \left(2 \psi \left(-\sqrt{4 \gamma^{2}+\cos ^{2}(2 \psi)}\right.\right.}{2 \gamma+\cos (2 \psi)+\sqrt{4 \gamma^{2}+\cos ^{2}(2 \psi)}}
\end{aligned}
$$

Since both of these expressions depend on $\psi$ at leading order in $K \ll 1$, the value of $C$, namely the ratio of vertical to horizontal displacements is not welldefined for either acoustic or optical mode. As in the case above, (3.24)-(3.25) give $U=V=0$ when $k=l=\pi$, and again (3.28)-(3.29) give divergences in $\widetilde{U}, \widetilde{V}$ (again, since $D=\mathcal{O}\left(K^{4}\right)$ by $(3.7)$ ).

\section{A.4 The Townes soliton and moving solutions of the $2 D N L S$}

Following Butt \& Wattis [8,9] we use the Townes soliton solution of the 2D NLS equation [11]. This solution is known to be unstable, with perturbations leading to blow-up or dissipation, as discussed by many authors, see Rasmussen \& Rypdal [26] and Fibich \& Papanicolaou [15] for more details. However, many perturbations lead to stable soliton solutions, for example, the fourth order system

$$
i F_{T}+D \nabla^{2} F+B|F|^{2} F+P \nabla^{4} F=0
$$

for $F(X, Y, T)$ studied by Karpman et al. [20] has stable soliton solutions when $P D<0$. The presence of an additional fifth-order nonlinearity can also stabilise the solitary wave solution, as in

$$
i F_{T}+D \nabla^{2} F+B|F|^{2} F+P \nabla^{4} F+K|F|^{4} F=0 .
$$

which Davydova et al. [13] showed has stable solutions when $P K>0$. Since our system (3.32) is merely the leading order approximation from an asymptotic expansion, we will also have fourth derivatives, fifth order nonlinearities and other terms at higher order in $\epsilon$, for example, second order spatial derivatives of cubic terms.

It is possible to 'boost' soliton solutions, by which we mean convert from a stationary breather to a moving breather through a generalisation of the solution. For example, in one spatial dimension, the stationary solution $F=$ $A \mathrm{e}^{-i B A^{2} T / 2} \operatorname{sech}(A X \sqrt{B / 2 D})$ of $i F_{T}=D F_{X X}+B|F|^{2} F$, can be generalised to

$$
F=A \exp \left(-\frac{i B A^{2} T}{2}-\frac{i V(2 X-V T)}{4 D}\right) \operatorname{sech}\left(A(X-V T) \sqrt{\frac{B}{2 D}}\right)
$$


Similarly, in two dimensions, the Townes soliton given by $\Phi(X, Y)$ which satisfies $\nabla^{2} \Phi=\Phi-\Phi^{3}$, and generates the static solution

$$
F=A \mathrm{e}^{-i B A^{2} T} \Phi(A X \sqrt{B / D}, A Y \sqrt{B / D})
$$

of $i F_{T}=D \nabla^{2} F+B|F|^{2} F$ can, by writing $Z=X-U T$ and $W=Y-V T$, be boosted to

$$
F=A \exp \left(-i B A^{2} T-\frac{i U(2 X-U T)+i V(2 Y-V T)}{4 D}\right) \Phi\left(A Z \sqrt{\frac{B}{D}}, A W \sqrt{\frac{B}{D}}\right)
$$

which also solves $i F_{T}=D \nabla^{2} F+B|F|^{2} F$.

\section{References}

[1] J.F.R. Archilla, N. Jiminez, V.J. Sanchez-Morcillo, L.M. Garcia-Raffi. Quodons in Mica. Nonlinear Localized Travelling Excitations in Crystals. Springer Series in Materials Science, vol 221. (2015).

[2] J. Bajars, J.C. Eilbeck, B. Leimkuhler. Numerical simulations of nonlinear modes in mica: past, present and future. In J.F.R Archilla, et al. Qudons in Mica. Springer Series in Materials Science, vol 221, (2015).

[3] J. Bajars, J.C. Eilbeck, B. Leimkuhler. Nonlinear propagating localized modes in a 2D hexagonal crystal lattice. Physica D, 301-302, 8-20, (2015).

[4] C.M. Bender, S. Orszag. Advanced Mathematical Methods for Scientists and Engineers. Springer, New York (1978).

[5] V. Besse H. Leblond, D. Mihalache, B.A. Malomed. Building patterns by traveling dipoles and vortices in two-dimensional periodic dissipative media. Opt. Commun., 332, 279-291, (2014).

[6] V. Besse, H. Leblond, D. Mihalache, B.A. Malomed. Pattern formation by traveling localized modes in two-dimensional dissipative media with lattice potentials. In J.F.R. Archilla, et al. Quodons in Mica. Springer Series in Materials Science, vol 221, (2015).

[7] I.A. Butt and J.A.D. Wattis. Asymptotic analysis of combined breather-kink modes in a Fermi-Pasta-Ulam chain, Physica D, 231, 165-179, (2007).

[8] I.A. Butt and J.A.D. Wattis. Discrete breathers in a two-dimensional FermiPasta-Ulam lattice. J. Phys. A; Math. Gen., 39, 4955-4984, (2006).

[9] I.A. Butt and J.A.D. Wattis. Discrete breathers in a two-dimensional hexagonal Fermi-Pasta-Ulam lattice. J. Phys. A; Math. Theor., 40, 1239-1264, (2007).

[10] A.P. Chetverikov, W. Ebeling, M.G. Verlade. Controlling fast electron transfer at the nanoscale by solitonic excitations along crystallographic axes. Euro. Phys. J. B 85, 291, (2012).

[11] R.Y. Chiao, E. Garmire \& C.H. Townes. Self-trapping of optical beams. Phys. Rev. Lett., 13, 479-, (1964). 
[12] L.A. Cisneros-Ake, A.A. Minzoni. A numerical study of weak lateral dispersion in discrete and continuum models. In J.F.R. Archilla, et al. Quodons in Mica. Springer Series in Materials Science, vol 221. (2015).

[13] T.A. Davydova, A.I. Yakimenko \& Y.A. Zaliznyak. Two-dimensional solitons and vortices in normal and anomalous dispersive media. Phys Rev E, 67, 026402, (2003).

[14] S.V. Dmitriev, A.A. Kistanov, V.I. Dubinko. Moving discrete breathers in 2D and 3D crystals. In J.F.R. Archilla, et al. Quodons in Mica. Springer Series in Materials Science, vol 221. (2015).

[15] G. Fibich \& G. Papanicolaou. Self-focusing in the perturbed and unperturbed nonlinear Schrödinger equation in critical dimension. SIAM J. Appl. Math., 60, 183, (1999).

[16] E. Fermi, J. Pasta, S. Ulam. (1955). Studies of Nonlinear Problems. Los Alamos Scientific Report, Document LA-1940. Reprinted in Lectures in Applied Mathematics. 15, 143-156, (1974).

[17] S. Flach, K. Kladko, R.S. MacKay. Energy thresholds for discrete breathers in one- two- and three-dimensional lattices. Phys. Rev. Lett., 78, 1207, (1997).

[18] G. Friesecke, R.L. Pego. Solitary waves on FPU lattices: I. Qualitative properties, renormalization and continuum limit, Nonlinearity, 12, 1601-27, (1999).

[19] M. Johansson, P. Jason. Breather mobility and the Peierls-Nabarro potential: brief review and recent progress. In J.F.R. Archilla, et al. Quodons in Mica. Springer Series in Materials Science, vol 221. 2015.

[20] V.I. Karpman. Stabilization of soliton instabilities by higher-order dispersion: fourth-order nonlinear Schrödinger-type equations. Phys. Rev. E, 53, R1336, (1996).

[21] A. Leonard, C. Chong, P.G. Kevrekidis, C. Daraio. Traveling waves in 2D hexagonal granular crystal lattices. Granular Matter, 16, 531, (2014).

[22] R.S. MacKay \& S. Aubry. Proof of existence of breathers for time-reversible or Hamiltonian networks of weakly coupled oscillators, Nonlinearity 7, 1623 (1994).

[23] J.L. Marin, J.C. Eilbeck, F.M. Russell. Breathers in cuprate-like lattices, Phys. Lett. A, 281, 21, (2001).

[24] J.L. Marin, J.C. Eilbeck, F.M. Russell. Localised moving breathers in a 2D hexagonal lattice, Phys. Lett. A, 248, 225 (1998).

[25] JD Murray. Asymptotic Analysis. Applied Mathematical Sciences, vol. 48. Springer, New York, (1984).

[26] J.J. Rasmussen \& K. Rypdal. Blow-up in nonlinear Schrödinger equations I: a general review. Physica Scripta, 33, 481-, (1989).

[27] M. Remoissenet. Low amplitude breather and envelope solitons in quasi-onedimensional physical models. Phys. Rev. B, 33, 2386-2392, (1985).

[28] N.F. Smyth. Modulation theory solution for resonant flow over topography. Proc. Roy. Soc. Lond. B Bio. 409, 79, (1987).

[29] S. Takeno, M. Peyrard. Nonlinear rotating modes: Green's-function solution. Phys. Rev. E, 55, 1922-1928, (1997).

[30] R.A. Vincencio, M. Johansson. Discrete soliton mobility in two-dimensional waveguide arrays with saturable nonlinearity. Phys. Rev. E 73, 046602, (2006)

[31] J.A.D. Wattis. Quasi-continuum approximations to lattice equations arising 
from the discrete nonlinear telegraph equation. J. Phys. A: Math. Gen., 33, 5925-5944, (2000).

[32] J.A.D. Wattis. Variational approximations to breathers in the discrete sineGordon equation II: moving breathers and Peierls-Nabarro energies. Nonlinearity, 9, 1583-1598, (1996).

[33] J.A.D. Wattis, L.M. James. Discrete breathers in honeycomb FPU lattices. J. Phys. A; Math. Theor., 47, 345101, (2014).

[34] X. Yi, J.A.D. Wattis, H. Susanto, L.J. Cummings. Discrete breathers in a twodimensional spring-mass lattice. J. Phys. A; Math. Theor., 42, 355207, (2009). 